J. Korean Math. Soc. 50 (2013), No. 2, pp. 275-306

http://dx.doi.org/10.4134/JKMS.2013.50.2.275

\title{
INVARIANT DIFFERENTIAL OPERATORS ON THE MINKOWSKI-EUCLID SPACE
}

\author{
JAE-HyUN YANG
}

\begin{abstract}
For two positive integers $m$ and $n$, let $\mathcal{P}_{n}$ be the open convex cone in $\mathbb{R}^{n(n+1) / 2}$ consisting of positive definite $n \times n$ real symmetric matrices and let $\mathbb{R}^{(m, n)}$ be the set of all $m \times n$ real matrices. In this paper, we investigate differential operators on the non-reductive homogeneous space $\mathcal{P}_{n} \times \mathbb{R}^{(m, n)}$ that are invariant under the natural action of the semidirect product group $G L(n, \mathbb{R}) \ltimes \mathbb{R}^{(m, n)}$ on the MinkowskiEuclid space $\mathcal{P}_{n} \times \mathbb{R}^{(m, n)}$. These invariant differential operators play an important role in the theory of automorphic forms on $G L(n, \mathbb{R}) \ltimes \mathbb{R}^{(m, n)}$ generalizing that of automorphic forms on $G L(n, \mathbb{R})$.
\end{abstract}

\section{Introduction}

Let

$$
\mathcal{P}_{n}=\left\{Y \in \mathbb{R}^{(n, n)} \mid Y={ }^{t} Y>0\right\}
$$

be the open convex cone of positive definite symmetric real matrices of degree $n$ in the Euclidean space $\mathbb{R}^{n(n+1) / 2}$, where $F^{(k, l)}$ denotes the set of all $k \times l$ matrices with entries in a commutative ring $F$ for two positive integers $k$ and $l$ and ${ }^{t} M$ denotes the transpose matrix of a matrix $M$. Then the general linear group $G L(n, \mathbb{R})$ acts on $\mathcal{P}_{n}$ transtively by

$$
g \cdot Y=g Y^{t} g, \quad g \in G L(n, \mathbb{R}), Y \in \mathcal{P}_{n} .
$$

Therefore, $\mathcal{P}_{n}$ is a symmetric space which is diffeomorphic to the quotient space $G L(n, \mathbb{R}) / O(n)$, where $O(n)$ denotes the orthogonal group of degree $n$. A. Selberg [10] investigated differential operators on $\mathcal{P}_{n}$ invariant under the action $(1.1)$ of $G L(n, \mathbb{R})$ (cf. [7, 8]).

Let

$$
G L_{n, m}=G L(n, \mathbb{R}) \ltimes \mathbb{R}^{(m, n)}
$$

Received January 7, 2012.

2010 Mathematics Subject Classification. Primary 13A50, 32Wxx, 15 A72.

Key words and phrases. invariants, invariant differential operators, the Minkowski-Euclid space.

This work was supported by Inha University Research Grant.

(C)2013 The Korean Mathematical Society 
be the semidirect product of $G L(n, \mathbb{R})$ and the abelian additive group $\mathbb{R}^{(m, n)}$ equipped with the following multiplication law

$$
(g, \lambda) \cdot(h, \mu)=\left(g h, \lambda^{t} h^{-1}+\mu\right),
$$

where $g, h \in G L(n, \mathbb{R})$ and $\lambda, \mu \in \mathbb{R}^{(m, n)}$. Then we have the natural action of $G L_{n, m}$ on the non-reductive homogeneous space $\mathcal{P}_{n} \times \mathbb{R}^{(m, n)}$ given by

$$
(g, \lambda) \cdot(Y, V)=\left(g Y^{t} g,(V+\lambda)^{t} g\right),
$$

where $g \in G L(n, \mathbb{R}), \lambda \in \mathbb{R}^{(m, n)}, Y \in \mathcal{P}_{n}$ and $V \in \mathbb{R}^{(m, n)}$.

For brevity, we set $\mathcal{P}_{n, m}=\mathcal{P}_{n} \times \mathbb{R}^{(m, n)}$ and $K=O(n)$. Since the action (1.2) of $G L_{n, m}$ is transitive, $\mathcal{P}_{n, m}$ is diffeomorphic to $G L_{n, m} / K$. We observe that the action (1.2) of $G L_{n, m}$ generalizes the action (1.1) of $G L(n, \mathbb{R})$.

The significance in studying the non-reductive homogeneous space $\mathcal{P}_{n, m}$ may be explained as follows. Let

$$
\Gamma_{n, m}=G L(n, \mathbb{Z}) \ltimes \mathbb{Z}^{(m, n)}
$$

be the arithmetic subgroup of $G L_{n, m}$, where $\mathbb{Z}$ is the ring of integers. The arithmetic quotient $\Gamma_{n, m} \backslash \mathcal{P}_{n, m}$ may be regarded as the universal family of principally polarized real tori of dimension $m n$ (cf. [14]). We propose to name the space $\mathcal{P}_{n, m}$ the Minkowski-Euclid space since it was H. Minkowski [9] who found a fundamental domain for $\mathcal{P}_{n}$ with respect to the arithmetic subgroup $G L(n, \mathbb{Z})$ by means of the reduction theory. In this setting, using the invariant differential operators on $\mathcal{P}_{n, m}$, we can develop a theory of automorphic forms on $\mathcal{P}_{n, m}$ generalizing that on $\mathcal{P}_{n}$.

The aim of this paper is to study differential operators on $\mathcal{P}_{n, m}$ that are invariant under the action (1.2) of $G L_{n, m}$. This paper is organized as follows. In Section 2, we review differential operators on $\mathcal{P}_{n}$ invariant under the action (1.1) of $G L(n, \mathbb{R})$. In Section 3, we investigate differential operators on $\mathcal{P}_{n, m}$ invariant under the action (1.2) of $G L_{n, m}$. For two positive integers $m$ and $n$, let

$$
S_{n, m}=\left\{(X, Z) \mid X={ }^{t} X \in \mathbb{R}^{(n, n)}, Z \in \mathbb{R}^{(m, n)}\right\}
$$

be the real vector space of dimension $\frac{n(n+1)}{2}+m n$. From the adjoint action of the group $G L_{n, m}$, we have the natural action of the orthogonal group $O(n)$ on $S_{n, m}$ given by

$$
k \cdot(X, Z)=\left(k X^{t} k, Z^{t} k\right), \quad k \in O(n),(X, Z) \in S_{n, m} .
$$

The action (1.3) of $K=O(n)$ induces canonically the representation $\sigma$ of $O(n)$ on the polynomial algebra $\operatorname{Pol}\left(S_{n, m}\right)$ consisting of complex-valued polynomial functions on $S_{n, m}$. Let $\operatorname{Pol}\left(S_{n, m}\right)^{K}$ denote the subalgebra of $\operatorname{Pol}\left(S_{n, m}\right)$ consisting of all polynomials on $S_{n, m}$ invariant under the representation $\sigma$ of $O(n)$, and $\mathbb{D}\left(\mathcal{P}_{n, m}\right)$ denote the algebra of all differential operators on $\mathcal{P}_{n, m}$ invariant under the action (1.2) of $G L_{n, m}$. We see that there is a canonically defined 
linear bijection of $\operatorname{Pol}\left(S_{n, m}\right)^{K}$ onto $\mathbb{D}\left(\mathcal{P}_{n, m}\right)$ which is not multiplicative. We will see that $\mathbb{D}\left(\mathcal{P}_{n, m}\right)$ is not commutative. The most important problem here is in finding a complete list of explicit generators of $\operatorname{Pol}\left(S_{n, m}\right)^{K}$ and a complete list of explicit generators of $\mathbb{D}\left(\mathcal{P}_{n, m}\right)$. We propose several natural problems. We present some explicit invariant differential operators which may be useful. In Section 4 , we deal with the case when $n=1$. In Section 5 , we deal with the case when $n=2$ and $m=1,2$. In Section 6 , we deal with the case when $n=3$ and $m=1,2$. In Section 7, we deal with the case when $n=4$ and $m=1,2$. In the final section, we present some open problems and discuss a notion of automorphic forms on $\mathcal{P}_{n, m}$ using $G L_{n, m}$-invariant differential operators on the Minkowski-Euclid space $\mathcal{P}_{n, m}$.

Acknowledgements. This work was in part done during the stay at the MaxPlanck-Institut für Mathematik in Bonn. The author is very grateful for the hospitality and financial support, and would like to give hearty and deep thanks to Minoru Itoh for his interest in this work and many fruitful discussions.

Notations. Denote by $\mathbb{Q}, \mathbb{R}$ and $\mathbb{C}$ the field of rational numbers, the field of real numbers and the field of complex numbers, respectively. Denote by $\mathbb{Z}$ and $\mathbb{Z}^{+}$the ring of integers and the set of all positive integers, respectively. The symbol ":=" means that the expression on the right is the definition of that on the left. For two positive integers $k$ and $l, F^{(k, l)}$ denotes the set of all $k \times l$ matrices with entries in a commutative ring $F$. For a square matrix $A \in F^{(k, k)}$ of degree $k, \operatorname{tr}(A)$ denotes the trace of $A$. For any $M \in F^{(k, l)},{ }^{t} M$ denotes the transposed matrix of $M$. For a positive integer $n, I_{n}$ denotes the identity matrix of degree $n$.

\section{Review on invariant differential operators on $\mathcal{P}_{n}$}

For a variable $Y=\left(y_{i j}\right) \in \mathcal{P}_{n}$, set

$$
d Y=\left(d y_{i j}\right) \text { and } \frac{\partial}{\partial Y}=\left(\frac{1+\delta_{i j}}{2} \frac{\partial}{\partial y_{i j}}\right),
$$

where $\delta_{i j}$ denotes the Kronecker delta symbol.

For a fixed element $g \in G L(n, \mathbb{R})$, put

$$
Y_{*}=g \cdot Y=g Y^{t} g, \quad Y \in \mathcal{P}_{n} .
$$

Then

$$
d Y_{*}=g d Y^{t} g \text { and } \quad \frac{\partial}{\partial Y_{*}}={ }^{t} g^{-1} \frac{\partial}{\partial Y} g^{-1} .
$$

Consider the following differential operators

$$
D_{i}=\operatorname{tr}\left(\left(Y \frac{\partial}{\partial Y}\right)^{i}\right), \quad i=1,2, \ldots, n,
$$


where $\operatorname{tr}(A)$ denotes the trace of a square matrix $A$. By Formula (2.1), we get

$$
\left(Y_{*} \frac{\partial}{\partial Y_{*}}\right)^{i}=g\left(Y \frac{\partial}{\partial Y}\right)^{i} g^{-1}
$$

for any $g \in G L(n, \mathbb{R})$. Hence each $D_{i}$ is invariant under the action (1.1) of $G L(n, \mathbb{R})$.

Selberg [10] proved the following.

Theorem 2.1. The algebra $\mathbb{D}\left(\mathcal{P}_{n}\right)$ of all differential operators on $\mathcal{P}_{n}$ invariant under the action $(1.1)$ of $G L(n, \mathbb{R})$ is generated by $D_{1}, D_{2}, \ldots, D_{n}$. Furthermore, $D_{1}, D_{2}, \ldots, D_{n}$ are algebraically independent and $\mathbb{D}\left(\mathcal{P}_{n}\right)$ is isomorphic to the commutative ring $\mathbb{C}\left[x_{1}, x_{2}, \ldots, x_{n}\right]$ with $n$ indeterminates $x_{1}, x_{2}, \ldots, x_{n}$.

Proof. The proof can be found in [4], p. 337, [8], pp. 64-66 and [11], pp. 29-30. The last statement follows immediately from the work of Harish-Chandra $[1,2]$ or [4], p. 294.

Let $\mathfrak{g}=\mathbb{R}^{(n, n)}$ be the Lie algebra of $G L(n, \mathbb{R})$. The adjoint representation Ad of $G L(n, \mathbb{R})$ is given by

$$
\operatorname{Ad}(g)=g X g^{-1}, \quad g \in G L(n, \mathbb{R}), X \in \mathfrak{g} .
$$

The Killing form $B$ of $\mathfrak{g}$ is given by

$$
B(X, Y)=2 n \operatorname{tr}(X Y)-2 \operatorname{tr}(X) \operatorname{tr}(Y), \quad X, Y \in \mathfrak{g} .
$$

Since $B\left(a I_{n}, X\right)=0$ for all $a \in \mathbb{R}$ and $X \in \mathfrak{g}, B$ is degenerate. So the Lie algebra $\mathfrak{g}$ of $G L(n, \mathbb{R})$ is not semi-simple.

The Lie algebra $\mathfrak{k}$ of $K$ is

$$
\mathfrak{k}=\left\{X \in \mathfrak{g} \mid X+{ }^{t} X=0\right\} .
$$

Let $\mathfrak{p}$ be the subspace of $\mathfrak{g}$ defined by

$$
\mathfrak{p}=\left\{X \in \mathfrak{g} \mid X={ }^{t} X \in \mathbb{R}^{(n, n)}\right\} .
$$

Then

$$
\mathfrak{g}=\mathfrak{k} \oplus \mathfrak{p}
$$

is the direct sum of $\mathfrak{k}$ and $\mathfrak{p}$ with respect to the Killing form $B$. Since $\operatorname{Ad}(k) \mathfrak{p} \subset \mathfrak{p}$ for any $k \in K, K$ acts on $\mathfrak{p}$ via the adjoint representation by

$$
k \cdot X=\operatorname{Ad}(k) X=k X^{t} k, \quad k \in K, X \in \mathfrak{p} .
$$

The action (2.3) induces the action of $K$ on the polynomial algebra $\operatorname{Pol}(\mathfrak{p})$ of $\mathfrak{p}$ and the symmetric algebra $S(\mathfrak{p})$. Denote by $\operatorname{Pol}(\mathfrak{p})^{K}\left(\operatorname{resp} ., S(\mathfrak{p})^{K}\right)$ the subalgebra of $\operatorname{Pol}(\mathfrak{p})$ (resp., $S(\mathfrak{p})$ ) consisting of all $K$-invariants. The following inner product $($,$) on \mathfrak{p}$ defined by

$$
(X, Y)=B(X, Y), \quad X, Y \in \mathfrak{p}
$$


gives an isomorphism as vector spaces

$$
\mathfrak{p} \cong \mathfrak{p}^{*}, \quad X \mapsto f_{X}, \quad X \in \mathfrak{p},
$$

where $\mathfrak{p}^{*}$ denotes the dual space of $\mathfrak{p}$ and $f_{X}$ is the linear functional on $\mathfrak{p}$ defined by

$$
f_{X}(Y)=(Y, X), \quad Y \in \mathfrak{p} .
$$

It is known that there is a canonical linear bijection of $S(\mathfrak{p})^{K}$ onto $\mathbb{D}\left(\mathcal{P}_{n}\right)$. Identifying $\mathfrak{p}$ with $\mathfrak{p}^{*}$ by the above isomorphism (2.4), we get a canonical linear bijection

$$
\Theta_{n}: \operatorname{Pol}(\mathfrak{p})^{K} \longrightarrow \mathbb{D}\left(\mathcal{P}_{n}\right)
$$

of $\operatorname{Pol}(\mathfrak{p})^{K}$ onto $\mathbb{D}\left(\mathcal{P}_{n}\right)$. The map $\Theta_{n}$ is described explicitly as follows. Put $N=n(n+1) / 2$. Let $\left\{\xi_{\alpha} \mid 1 \leq \alpha \leq N\right\}$ be a basis of $\mathfrak{p}$. If $P \in \operatorname{Pol}(\mathfrak{p})^{K}$, then

$$
\left(\Theta_{n}(P) f\right)(g K)=\left[P\left(\frac{\partial}{\partial t_{\alpha}}\right) f\left(g \exp \left(\sum_{\alpha=1}^{N} t_{\alpha} \xi_{\alpha}\right) K\right)\right]_{\left(t_{\alpha}\right)=0},
$$

where $f \in C^{\infty}\left(\mathcal{P}_{n}\right)$. We refer the reader to $[3,4]$ for more detail. In general, it is difficult to express $\Theta_{n}(P)$ explicitly for a polynomial $P \in \operatorname{Pol}(\mathfrak{p})^{K}$.

Let

$$
q_{i}(X)=\operatorname{tr}\left(X^{i}\right), \quad i=1,2, \ldots, n
$$

be the polynomials on $\mathfrak{p}$. Here we take coordinates $x_{11}, x_{12}, \ldots, x_{n n}$ in $\mathfrak{p}$ given by

$$
X=\left(\begin{array}{cccc}
x_{11} & \frac{1}{2} x_{12} & \ldots & \frac{1}{2} x_{1 n} \\
\frac{1}{2} x_{12} & x_{22} & \ldots & \frac{1}{2} x_{2 n} \\
\vdots & \vdots & \ddots & \vdots \\
\frac{1}{2} x_{1 n} & \frac{1}{2} x_{2 n} & \ldots & x_{n n}
\end{array}\right)
$$

For any $k \in K$,

$$
\left(k \cdot q_{i}\right)(X)=q_{i}\left(k^{-1} X k\right)=\operatorname{tr}\left(k^{-1} X^{i} k\right)=q_{i}(X), \quad i=1,2, \ldots, n .
$$

Thus $q_{i} \in \operatorname{Pol}(\mathfrak{p})^{K}$ for $i=1,2, \ldots, n$. By a classical invariant theory (cf. $[5,12])$, we can prove that the algebra $\operatorname{Pol}(\mathfrak{p})^{K}$ is generated by the polynomials $q_{1}, q_{2}, \ldots, q_{n}$ and that $q_{1}, q_{2}, \ldots, q_{n}$ are algebraically independent. Using Formula (2.6), we can show without difficulty that

$$
\Theta_{n}\left(q_{1}\right)=\operatorname{tr}\left(2 Y \frac{\partial}{\partial Y}\right)
$$

However, $\Theta_{n}\left(q_{i}\right)(i=2,3, \ldots, n)$ are yet known explicitly.

We propose the following conjecture. 
Conjecture 1. For any $n$,

$$
\Theta_{n}\left(q_{i}\right)=\operatorname{tr}\left(\left(2 Y \frac{\partial}{\partial Y}\right)^{i}\right), \quad i=1,2, \ldots, n .
$$

Remark. The author has verified that the above conjecture is true for $n=1,2$.

For a positive real number $A$,

$$
d s_{n ; A}^{2}=A \cdot \operatorname{tr}\left(Y^{-1} d Y Y^{-1} d Y\right)
$$

is a Riemannian metric on $\mathcal{P}_{n}$ invariant under the action (1.1). The Laplacian $\Delta_{n ; A}$ of $d s_{n ; A}^{2}$ is given by

$$
\Delta_{n ; A}=\frac{1}{A} \operatorname{tr}\left(\left(Y \frac{\partial}{\partial Y}\right)^{2}\right) .
$$

For instance, consider the case when $n=2$ and $A>0$. If we write for $Y \in \mathcal{P}_{2}$,

$$
Y=\left(\begin{array}{ll}
y_{1} & y_{3} \\
y_{3} & y_{2}
\end{array}\right) \quad \text { and } \quad \frac{\partial}{\partial Y}=\left(\begin{array}{cc}
\frac{\partial}{\partial y_{1}} & \frac{1}{2} \frac{\partial}{\partial y_{3}} \\
\frac{1}{2} \frac{\partial}{\partial y_{3}} & \frac{\partial}{\partial y_{2}}
\end{array}\right)
$$

then

$$
\begin{aligned}
d s_{2 ; A}^{2}= & A \operatorname{tr}\left(Y^{-1} d Y Y^{-1} d Y\right) \\
= & \frac{A}{\left(y_{1} y_{2}-y_{3}^{2}\right)^{2}}\left\{y_{2}^{2} d y_{1}^{2}+y_{1}^{2} d y_{2}^{2}+2\left(y_{1} y_{2}+y_{3}^{2}\right) d y_{3}^{2}\right. \\
& \left.\quad+2 y_{3}^{2} d y_{1} d y_{2}-4 y_{2} y_{3} d y_{1} d y_{3}-4 y_{1} y_{3} d y_{2} d y_{3}\right\}
\end{aligned}
$$

and its Laplacian $\Delta_{2 ; A}$ on $\mathcal{P}_{2}$ is

$$
\begin{aligned}
\Delta_{2 ; A}=\frac{1}{A} & \operatorname{tr}\left(\left(Y \frac{\partial}{\partial Y}\right)^{2}\right) \\
= & \frac{1}{A}\left\{y_{1}^{2} \frac{\partial^{2}}{\partial y_{1}^{2}}+y_{2}^{2} \frac{\partial^{2}}{\partial y_{2}^{2}}+\frac{1}{2}\left(y_{1} y_{2}+y_{3}^{2}\right) \frac{\partial^{2}}{\partial y_{3}^{2}}\right. \\
& +2\left(y_{3}^{2} \frac{\partial^{2}}{\partial y_{1} \partial y_{2}}+y_{1} y_{3} \frac{\partial^{2}}{\partial y_{1} \partial y_{3}}+y_{2} y_{3} \frac{\partial^{2}}{\partial y_{2} \partial y_{3}}\right) \\
& \left.+\frac{3}{2}\left(y_{1} \frac{\partial}{\partial y_{1}}+y_{2} \frac{\partial}{\partial y_{2}}+y_{3} \frac{\partial}{\partial y_{3}}\right)\right\} .
\end{aligned}
$$

3. Invariant differential operators on $\mathcal{P}_{n, m}$

For a variable $(Y, V) \in \mathcal{P}_{n, m}$ with $Y \in \mathcal{P}_{n}$ and $V \in \mathbb{R}^{(m, n)}$, put

$$
\begin{gathered}
Y=\left(y_{i j}\right) \text { with } y_{i j}=y_{j i}, \quad V=\left(v_{k l}\right), \\
d Y=\left(d y_{i j}\right), \quad d V=\left(d v_{k l}\right),
\end{gathered}
$$


and

$$
[d Y]=\wedge_{i \leq j} d y_{i j}, \quad[d V]=\wedge_{k, l} d v_{k l},
$$

$$
\frac{\partial}{\partial Y}=\left(\frac{1+\delta_{i j}}{2} \frac{\partial}{\partial y_{i j}}\right), \quad \frac{\partial}{\partial V}=\left(\frac{\partial}{\partial v_{k l}}\right)
$$

where $1 \leq i, j, l \leq n$ and $1 \leq k \leq m$.

For a fixed element $(g, \lambda) \in G L_{n, m}$, write

$$
\left(Y_{\star}, V_{\star}\right)=(g, \lambda) \cdot(Y, V)=\left(g Y^{t} g,(V+\lambda)^{t} g\right),
$$

where $(Y, V) \in \mathcal{P}_{n, m}$. Then we get

$$
Y_{\star}=g Y^{t} g, \quad V_{\star}=(V+\lambda)^{t} g
$$

$$
\frac{\partial}{\partial Y_{\star}}={ }^{t} g^{-1} \frac{\partial}{\partial Y} g^{-1}, \quad \frac{\partial}{\partial V_{\star}}=\frac{\partial}{\partial V} g^{-1} .
$$

Lemma 3.1. For any two positive real numbers $A$ and $B$, the following metric $d s_{n, m ; A, B}^{2}$ on $\mathcal{P}_{n, m}$ defined by

$$
d s_{n, m ; A, B}^{2}=A \sigma\left(Y^{-1} d Y Y^{-1} d Y\right)+B \sigma\left(Y^{-1 t}(d V) d V\right)
$$

is a Riemannian metric on $\mathcal{P}_{n, m}$ which is invariant under the action (1.2) of $G L_{n, m}$. The Laplacian $\Delta_{n, m ; A, B}$ of $\left(\mathcal{P}_{n, m}, d s_{n, m ; A, B}^{2}\right)$ is given by

$\Delta_{n, m ; A, B}=\frac{1}{A} \sigma\left(\left(Y \frac{\partial}{\partial Y}\right)^{2}\right)-\frac{m}{2 A} \sigma\left(Y \frac{\partial}{\partial Y}\right)+\frac{1}{B} \sum_{k \leq p}\left(\left(\frac{\partial}{\partial V}\right) Y^{t}\left(\frac{\partial}{\partial V}\right)\right)_{k p}$.

Moreover, $\Delta_{n, m ; A, B}$ is a differential operator of order 2 which is invariant under the action (1.2) of $G L_{n, m}$.

Proof. The proof can be found in [14].

Lemma 3.2. The following volume element $d v_{n, m}(Y, V)$ on $\mathcal{P}_{n, m}$ defined by

$$
d v_{n, m}(Y, V)=(\operatorname{det} Y)^{-\frac{n+m+1}{2}}[d Y][d V]
$$

is invariant under the action (1.2) of $G L_{n, m}$.

Proof. The proof can be found in [14].

Theorem 3.1. Any geodesic through the origin $\left(I_{n}, 0\right)$ for the Riemannian metric $d s_{n, m ; 1,1}^{2}$ is of the form

$$
\gamma(t)=\left(\lambda(2 t)[k], Z\left(\int_{0}^{t} \lambda(t-s) d s\right)[k]\right),
$$

where $k$ is a fixed element of $O(n), Z$ is a fixed $h \times g$ real matrix, $t$ is a real variable, $\lambda_{1}, \lambda_{2}, \ldots, \lambda_{n}$ are fixed real numbers not all zero and

$$
\lambda(t):=\operatorname{diag}\left(e^{\lambda_{1} t}, \ldots, e^{\lambda_{n} t}\right) .
$$

Furthermore, the tangent vector $\gamma^{\prime}(0)$ of the geodesic $\gamma(t)$ at $\left(I_{n}, 0\right)$ is $(D[k], Z)$, where $D=\operatorname{diag}\left(2 \lambda_{1}, \ldots, 2 \lambda_{n}\right)$. 
Proof. The proof can be found in [14].

Theorem 3.2. Let $\left(Y_{0}, V_{0}\right)$ and $\left(Y_{1}, V_{1}\right)$ be two points in $\mathcal{P}_{n, m}$. Let $g$ be an element in $G L(n, \mathbb{R})$ such that $Y_{0}\left[{ }^{t} g\right]=I_{n}$ and $Y_{1}\left[{ }^{t} g\right]$ is diagonal. Then the length $s\left(\left(Y_{0}, V_{0}\right),\left(Y_{1}, V_{1}\right)\right)$ of the geodesic joining $\left(Y_{0}, V_{0}\right)$ and $\left(Y_{1}, V_{1}\right)$ for the $G L_{n, m}$-invariant Riemannian metric $d s_{n, m ; A, B}^{2}$ is given by

$s\left(\left(Y_{0}, V_{0}\right),\left(Y_{1}, V_{1}\right)\right)=A\left\{\sum_{j=1}^{n}\left(\ln t_{j}\right)^{2}\right\}^{1 / 2}+B \int_{0}^{1}\left(\sum_{j=1}^{n} \Delta_{j} e^{-\left(\ln t_{j}\right) t}\right)^{1 / 2} d t$,

where $\Delta_{j}=\sum_{k=1}^{m} \widetilde{v}_{k j}^{2}(1 \leq j \leq n)$ with $\left(V_{1}-V_{0}\right)^{t} g=\left(\widetilde{v}_{k j}\right)$ and $t_{1}, \ldots, t_{n}$ denotes the zeros of $\operatorname{det}\left(t Y_{0}-Y_{1}\right)$.

Proof. The proof can be found in [14].

The Lie algebra $\mathfrak{g}_{\star}$ of $G L_{n, m}$ is given by

$$
\mathfrak{g}_{\star}=\left\{(X, Z) \mid X \in \mathbb{R}^{(n, n)}, Z \in \mathbb{R}^{(m, n)}\right\}
$$

equipped with the following Lie bracket

$$
\left[\left(X_{1}, Z_{1}\right),\left(X_{2}, Z_{2}\right)\right]=\left(\left[X_{1}, X_{2}\right]_{0}, Z_{2}{ }^{t} X_{1}-Z_{1}{ }^{t} X_{2}\right),
$$

where $\left[X_{1}, X_{2}\right]_{0}=X_{1} X_{2}-X_{2} X_{1}$ denotes the usual matrix bracket and $\left(X_{1}, Z_{1}\right)$, $\left(X_{2}, Z_{2}\right) \in \mathfrak{g}_{\star}$. The adjoint representation $\mathrm{Ad}_{\star}$ of $G L_{n, m}$ is given by

$$
\operatorname{Ad}_{\star}((g, \lambda))(X, Z)=\left(g X g^{-1},\left(Z-\lambda^{t} X\right)^{t} g\right),
$$

where $(g, \lambda) \in G L_{n, m}$ and $(X, Z) \in \mathfrak{g}_{\star}$. Also, the adjoint representation $\operatorname{ad}_{\star}$ of $\mathfrak{g}_{\star}$ on End $\left(\mathfrak{g}_{\star}\right)$ is given by

$$
\operatorname{ad}_{\star}((X, Z))\left(\left(X_{1}, Z_{1}\right)\right)=\left[(X, Z),\left(X_{1}, Z_{1}\right)\right] .
$$

We see that the Killing form $B_{\star}$ of $\mathfrak{g}_{\star}$ is given by

$$
B_{\star}\left(\left(X_{1}, Z_{1}\right),\left(X_{2}, Z_{2}\right)\right)=(2 n+m) \operatorname{tr}\left(X_{1} X_{2}\right)-2 \operatorname{tr}\left(X_{1}\right) \operatorname{tr}\left(X_{2}\right) .
$$

The Lie algebra $\mathfrak{k}$ of $K$ is

$$
\mathfrak{k}=\left\{(X, 0) \in \mathfrak{g}_{\star} \mid X+{ }^{t} X=0\right\} .
$$

Let $\mathfrak{p}_{\star}$ be the subspace of $\mathfrak{g}_{\star}$ defined by

$$
\mathfrak{p}_{\star}=\left\{(X, Z) \in \mathfrak{g}_{\star} \mid X={ }^{t} X \in \mathbb{R}^{(n, n)}, Z \in \mathbb{R}^{(m, n)}\right\} .
$$

Then we have the following relations

$$
[\mathfrak{k}, \mathfrak{k}] \subset \mathfrak{k} \quad \text { and } \quad\left[\mathfrak{k}, \mathfrak{p}_{\star}\right] \subset \mathfrak{p}_{\star} .
$$

In addition, we have

$$
\left.\mathfrak{g}_{\star}=\mathfrak{k} \oplus \mathfrak{p}_{\star} \quad \text { ( the direct sum }\right) .
$$


$K$ acts on $\mathfrak{p}_{\star}$ via the adjoint representation $\mathrm{Ad}_{\star}$ of $G L_{n, m}$ by

$$
k \cdot(X, Z)=\left(k X^{t} k, Z^{t} k\right), \quad k \in K,(X, Z) \in \mathfrak{p}_{\star} .
$$

The action (3.7) induces the action of $K$ on the polynomial algebra $\operatorname{Pol}\left(\mathfrak{p}_{\star}\right)$ of $\mathfrak{p}_{\star}$ and the symmetric algebra $S\left(\mathfrak{p}_{\star}\right)$. Denote by $\operatorname{Pol}\left(\mathfrak{p}_{\star}\right)^{K}\left(\operatorname{resp} ., S\left(\mathfrak{p}_{\star}\right)^{K}\right)$ the subalgebra of $\operatorname{Pol}\left(\mathfrak{p}_{\star}\right)$ (resp., $S\left(\mathfrak{p}_{\star}\right)$ ) consisting of all $K$-invariants. The following inner product $(,)_{\star}$ on $\mathfrak{p}_{\star}$ defined by

$$
\left(\left(X_{1}, Z_{1}\right),\left(X_{2}, Z_{2}\right)\right)_{\star}=\operatorname{tr}\left(X_{1} X_{2}\right)+\operatorname{tr}\left(Z_{1}{ }^{t} Z_{2}\right), \quad\left(X_{1}, Z_{1}\right),\left(X_{2}, Y_{2}\right) \in \mathfrak{p}_{\star}
$$

gives an isomorphism as vector spaces

$$
\mathfrak{p}_{\star} \cong \mathfrak{p}_{\star}^{*}, \quad(X, Z) \mapsto f_{X, Z}, \quad(X, Z) \in \mathfrak{p}_{\star},
$$

where $\mathfrak{p}_{\star}^{*}$ denotes the dual space of $\mathfrak{p}_{\star}$ and $f_{X, Z}$ is the linear functional on $\mathfrak{p}_{\star}$ defined by

$$
f_{X, Z}\left(\left(X_{1}, Z_{1}\right)\right)=\left((X, Z),\left(X_{1}, Z_{1}\right)\right)_{\star}, \quad\left(X_{1}, Z_{1}\right) \in \mathfrak{p}_{\star} .
$$

Let $\mathbb{D}\left(\mathcal{P}_{n, m}\right)$ be the algebra of all differential operators on $\mathcal{P}_{n, m}$ that are invariant under the action (1.2) of $G L_{n, m}$. It is known that there is a canonical linear bijection of $S\left(\mathfrak{p}_{\star}\right)^{K}$ onto $\mathbb{D}\left(\mathcal{P}_{n, m}\right)$. Identifying $\mathfrak{p}_{\star}$ with $\mathfrak{p}_{\star}^{*}$ by the above isomorphism (3.5), we get a canonical linear bijection

$$
\Theta_{n, m}: \operatorname{Pol}\left(\mathfrak{p}_{\star}\right)^{K} \longrightarrow \mathbb{D}\left(\mathcal{P}_{n, m}\right)
$$

of $\operatorname{Pol}\left(\mathfrak{p}_{\star}\right)^{K}$ onto $\mathbb{D}\left(\mathcal{P}_{n, m}\right)$. The map $\Theta_{n, m}$ is described explicitly as follows. Put $N_{\star}=n(n+1) / 2+m n$. Let $\left\{\eta_{\alpha} \mid 1 \leq \alpha \leq N_{\star}\right\}$ be a basis of $\mathfrak{p}_{\star}$. If $P \in \operatorname{Pol}\left(\mathfrak{p}_{\star}\right)^{K}$, then

$$
\left(\Theta_{n, m}(P) f\right)(g K)=\left[P\left(\frac{\partial}{\partial t_{\alpha}}\right) f\left(g \exp \left(\sum_{\alpha=1}^{N_{\star}} t_{\alpha} \eta_{\alpha}\right) K\right)\right]_{\left(t_{\alpha}\right)=0},
$$

where $f \in C^{\infty}\left(\mathcal{P}_{n, m}\right)$. We refer the reader to [4], pp. 280-289. In general, it is very hard to express $\Theta_{n, m}(P)$ explicitly for a polynomial $P \in \operatorname{Pol}\left(\mathfrak{p}_{\star}\right)^{K}$.

Take a coordinate $(X, Z)$ in $\mathfrak{p}_{\star}$ such that

$$
X=\left(\begin{array}{cccc}
x_{11} & \frac{1}{2} x_{12} & \ldots & \frac{1}{2} x_{1 n} \\
\frac{1}{2} x_{12} & x_{22} & \ldots & \frac{1}{2} x_{2 n} \\
\vdots & \vdots & \ddots & \vdots \\
\frac{1}{2} x_{1 n} & \frac{1}{2} x_{2 n} & \ldots & x_{n n}
\end{array}\right) \in \mathfrak{p} \quad \text { and } \quad Z=\left(z_{k l}\right) \in \mathbb{R}^{(m, n)}
$$

Define the polynomials $\alpha_{j}, \beta_{p q}^{(k)}, R_{j p}$ and $S_{j p}$ on $\mathfrak{p}_{\star}$ by

$$
\begin{aligned}
(3.11) \alpha_{j}(X, Z) & =\operatorname{tr}\left(X^{j}\right), \quad 1 \leq j \leq n, \\
(3.12) \beta_{p q}^{(k)}(X, Z) & =\left(Z X^{k t} Z\right)_{p q}, \quad 0 \leq k \leq n-1,1 \leq p \leq q \leq m, \\
(3.13) R_{j p}(X, Z) & =\operatorname{tr}\left(X^{j}\left({ }^{t} Z Z\right)^{p}\right), \quad 0 \leq j \leq n-1,1 \leq p \leq m, \\
(3.14) S_{j p}(X, Z) & =\operatorname{det}\left(X^{j}\left({ }^{t} Z Z\right)^{p}\right), \quad 0 \leq j \leq n-1,1 \leq p \leq m,
\end{aligned}
$$


where $\left(Z^{t} Z\right)_{p q}\left(\right.$ resp., $\left.\left(Z X^{t} Z\right)_{p q}\right)$ denotes the $(p, q)$-entry of $Z^{t} Z$ (resp., $\left.Z X^{t} Z\right)$.

For any $m \times m$ real matrix $S$, define the polynomials $M_{j ; S}, Q_{p ; S}, \Omega_{i, p, j ; S}$ and $\Theta_{i, p, j ; S}$ on $\mathfrak{p}_{\star}$ by

$$
\begin{aligned}
M_{j ; S}(X, Z) & =\operatorname{tr}\left(\left(X+{ }^{t} Z S Z\right)^{j}\right), \quad 1 \leq j \leq n, \\
Q_{p ; S}(X, Z) & =\operatorname{tr}\left(\left({ }^{t} Z S Z\right)^{p}\right), \quad 1 \leq p \leq n, \\
\Omega_{i, p, j ; S}(X, Z) & =\operatorname{tr}\left(X^{i}\left({ }^{t} Z S Z\right)^{p}\left(X+{ }^{t} Z S Z\right)^{j}\right), \\
\Theta_{i, p, j ; S}(X, Z) & =\operatorname{det}\left(X^{i}\left({ }^{t} Z S Z\right)^{p}\left(X+{ }^{t} Z S Z\right)^{j}\right),
\end{aligned}
$$

where $0 \leq i, j \leq n-1,1 \leq p \leq n$. We see that all $\alpha_{j}, \beta_{p q}^{(k)}, R_{j p}, S_{j p}, M_{j ; S}$, $Q_{p ; S}, \Omega_{i, p, j ; S}$ and $\Theta_{i, p, j ; S}$ are elements of $\operatorname{Pol}\left(\mathfrak{p}_{\star}\right)^{K}$.

We propose the following natural problems.

Problem 1. Find a complete list of explicit generators of $\operatorname{Pol}\left(\mathfrak{p}_{\star}\right)^{K}$.

Problem 2. Find all relations among a set of generators of $\operatorname{Pol}\left(\mathfrak{p}_{\star}\right)^{K}$.

Problem 3. Find an easy or an effective way to express explicitly the images of the above invariant polynomials under the Helgason map $\Theta_{n, m}$.

Problem 4. Decompose $\operatorname{Pol}\left(\mathfrak{p}_{\star}\right)^{K}$ into $O(n)$-irreducibles.

Problem 5. Find a complete list of explicit generators of the algebra $\mathbb{D}\left(\mathcal{P}_{n, m}\right)$ or construct explicit $G L_{n, m}$-invariant differential operators on $\mathcal{P}_{n, m}$.

Problem 6. Find all relations among a set of generators of $\mathbb{D}\left(\mathcal{P}_{n, m}\right)$.

Problem 7. Is $\operatorname{Pol}\left(\mathfrak{p}_{\star}\right)^{K}$ finitely generated? Is $\mathbb{D}\left(\mathcal{P}_{n, m}\right)$ finitely generated?

M. Itoh [6] proved the following theorem.

Theorem 3.3. Pol $\left(\mathfrak{p}_{\star}\right)^{K}$ is generated by $\alpha_{j}(1 \leq j \leq n)$ and $\beta_{p q}^{(k)}(0 \leq k \leq$ $n-1,1 \leq p \leq q \leq m)$.

Proof. We refer the reader to Theorem 3.1 in [6].

M. Itoh solved Problem 2 in [6], Theorem 3.2.

We present some invariant differential operators on $\mathcal{P}_{n, m}$. Define the differential operators $D_{j}, \Omega_{p q}$ and $L_{p}$ on $\mathcal{P}_{n, m}$ by

$$
D_{j}=\operatorname{tr}\left(\left(2 Y \frac{\partial}{\partial Y}\right)^{j}\right), \quad 1 \leq j \leq n,
$$

$$
\Omega_{p q}^{(k)}=\left\{\frac{\partial}{\partial V}\left(2 Y \frac{\partial}{\partial Y}\right)^{k} Y^{t}\left(\frac{\partial}{\partial V}\right)\right\}_{p q}, \quad 0 \leq k \leq n-1,1 \leq p \leq q \leq m
$$


and

$$
L_{p}=\operatorname{tr}\left(\left\{Y^{t}\left(\frac{\partial}{\partial V}\right) \frac{\partial}{\partial V}\right\}^{p}\right), \quad 1 \leq p \leq m .
$$

Here, for a matrix $A$, we denote by $A_{p q}$ the $(p, q)$-entry of $A$.

Also, define the differential operators $S_{j p}$ by

$$
S_{j p}=\operatorname{tr}\left(\left(2 Y \frac{\partial}{\partial Y}\right)^{j}\left\{Y^{t}\left(\frac{\partial}{\partial V}\right) \frac{\partial}{\partial V}\right\}^{p}\right),
$$

where $1 \leq j \leq n$ and $1 \leq p \leq m$.

For any real matrix $S$ of degree $m$, define the differential operators $\Phi_{j ; S}, L_{p ; S}$ and $\Phi_{i, p, j ; S}$ by

$$
\begin{gathered}
\Phi_{j ; S}=\operatorname{tr}\left(\left\{Y\left(2 \frac{\partial}{\partial Y}+{ }^{t}\left(\frac{\partial}{\partial V}\right) S\left(\frac{\partial}{\partial V}\right)\right)\right\}^{j}\right), \quad 1 \leq j \leq n, \\
L_{p ; S}=\operatorname{tr}\left(\left\{Y^{t}\left(\frac{\partial}{\partial V}\right) S\left(\frac{\partial}{\partial V}\right)\right\}^{p}\right), \quad 1 \leq p \leq m
\end{gathered}
$$

and

$$
\begin{aligned}
& \Phi_{i, p, j ; S}(X, Z) \\
= & \operatorname{tr}\left(\left(2 Y \frac{\partial}{\partial Y}\right)^{i}\left(Y^{t}\left(\frac{\partial}{\partial V}\right) S\left(\frac{\partial}{\partial V}\right)\right)^{p}\left\{Y\left(2 \frac{\partial}{\partial Y}+{ }^{t}\left(\frac{\partial}{\partial V}\right) S\left(\frac{\partial}{\partial V}\right)\right)\right\}^{j}\right) .
\end{aligned}
$$

We want to mention a special invariant differential operator on $\mathcal{P}_{n, m}$. In [13], the author studied the following differential operator $M_{n, m, \mathcal{M}}$ on $\mathcal{P}_{n, m}$ defined by

$$
M_{n, m, \mathcal{M}}=\operatorname{det}(Y) \cdot \operatorname{det}\left(\frac{\partial}{\partial Y}+\frac{1}{8 \pi}^{t}\left(\frac{\partial}{\partial V}\right) \mathcal{M}^{-1}\left(\frac{\partial}{\partial V}\right)\right),
$$

where $\mathcal{M}$ is a positive definite, symmetric half-integral matrix of degree $m$. This differential operator characterizes singular Jacobi forms. For more detail, we refer the reader to [13]. From (3.1) and (3.2), we can easily see that the differential operator $M_{n, m, \mathcal{M}}$ is invariant under the action (1.2) of $G L_{n, m}$.

Question. Calculate the inverse of $M_{n, m, \mathcal{M}}$ under the Helgason map $\Theta_{n, m}$.

\section{The case when $n=1$}

In this section, we consider the case when $n=m=1$ and the case when $n=1$ and $m \geq 2$ separately.

\subsection{The case when $n=1$ and $m=1$}


In this case,

$$
G L_{1,1}=\mathbb{R}^{\times} \ltimes \mathbb{R}, \quad K=O(1), \quad \mathcal{P}_{1,1}=\mathbb{R}^{+} \times \mathbb{R},
$$

where $\mathbb{R}^{\times}=\{a \in \mathbb{R} \mid a \neq 0\}$ and $\mathbb{R}^{+}=\{a \in \mathbb{R} \mid a>0\}$. Clearly, $\mathfrak{k}=0$ and $\mathfrak{p}_{\star}=\mathfrak{g}_{\star}=\{(x, z) \mid x, z \in \mathbb{R}\}$. Then $e=(1,0)$ and $f=(0,1)$ form the standard basis for $\mathfrak{p}_{\star}$. Using this basis, we take a coordinate $(x, z)$ in $\mathfrak{p}_{\star}$; that is, if $w \in \mathfrak{p}_{\star}$, then we write $w=x e+z f$. We can show that $\operatorname{Pol}\left(\mathfrak{p}_{\star}\right)^{K}$ is generated by the following polynomials

$$
\alpha(x, z)=x \quad \text { and } \quad \beta(x, z)=z^{2} .
$$

The generators $\alpha$ and $\beta$ are algebraically independent. Let $(y, v)$ be a coordinate in $\mathcal{P}_{1,1}$ with $y>0$ and $v \in \mathbb{R}$. Then using Formula (3.10), we can show that

$$
\Theta_{1,1}(\alpha)=2 y \frac{\partial}{\partial y} \quad \text { and } \quad \Theta_{1,1}(\beta)=y \frac{\partial^{2}}{\partial v^{2}} \text {. }
$$

We see that $\Theta_{1,1}(\alpha)$ and $\Theta_{1,1}(\beta)$ generate the algebra $\mathbb{D}\left(\mathcal{P}_{1,1}\right)$ and are algebraically dependent. Indeed, we have the following noncommutation relation

$$
\Theta_{1,1}(\alpha) \Theta_{1,1}(\beta)-\Theta_{1,1}(\beta) \Theta_{1,1}(\alpha)=2 \Theta_{1,1}(\beta) .
$$

Hence the algebra $\mathbb{D}\left(\mathcal{P}_{1,1}\right)$ is not commutative. The unitary dual $\widehat{K}$ of $K$ consists of two elements. Let

$$
\operatorname{Pol}\left(\mathfrak{p}_{\star}\right)=\sum_{\tau \in \widehat{K}} m_{\tau} \tau
$$

be the decomposition of $\operatorname{Pol}\left(\mathfrak{p}_{\star}\right)$ into $K$-irreducibles. It is easy to see that the multiplicity $m_{\tau}$ of $\tau$ is infinite for all $\tau \in \widehat{K}$. So the action of $K$ on $\operatorname{Pol}\left(\mathfrak{p}_{\star}\right)$ is not multiplicity-free. In this case, the seven problems proposed in Section 3 are completely solved.

\subsection{The case when $n=1$ and $m \geq 2$}

Consider the case when $n=1$ and $m \geq 2$. In this case,

$$
G L_{1, m}=\mathbb{R}^{\times} \ltimes \mathbb{R}^{(m, 1)}, \quad K=O(1), \quad \mathcal{P}_{1, m}=\mathbb{R}^{+} \times \mathbb{R}^{(m, 1)},
$$

where $\mathbb{R}^{\times}=\{a \in \mathbb{R} \mid a \neq 0\}$ and $\mathbb{R}^{+}=\{a \in \mathbb{R} \mid a>0\}$. Clearly, $\mathfrak{k}=0$ and $\mathfrak{p}_{\star}=\mathfrak{g}_{\star}=\left\{(x, z) \mid x \in \mathbb{R}, z \in \mathbb{R}^{(m, 1)}\right\}$. Let $\left\{e_{1}, \ldots, e_{m}\right\}$ be the standard basis of $\mathbb{R}^{(m, 1)}$. Then

$$
\eta_{0}=(1,0), \eta_{1}=\left(0, e_{1}\right), \eta_{2}=\left(0, e_{2}\right), \ldots, \eta_{m}=\left(0, e_{m}\right)
$$

form a basis of $\mathfrak{p}_{\star}$. Using this basis, we take a coordinate $\left(x, z_{1}, z_{2}, \ldots, z_{m}\right)$ in $\mathfrak{p}_{\star}$; that is, if $w \in \mathfrak{p}_{\star}$, then we write $w=x \eta_{0}+\sum_{k=1}^{m} z_{k} \eta_{k}$. We can show that $\operatorname{Pol}\left(\mathfrak{p}_{\star}\right)^{K}$ is generated by the following polynomials

$$
\alpha(x, z)=x \quad \text { and } \quad \beta_{k l}(x, z)=z_{k} z_{l}, \quad 1 \leq k \leq l \leq m
$$

where $z=\left(z_{1}, z_{2}, \ldots, z_{m}\right)$. We see easily that one has the following relations

$$
\beta_{k k} \beta_{l l}=\beta_{k l}^{2} \quad \text { for } 1 \leq k<l \leq m
$$


and

$$
\beta_{k k} \beta_{l l}^{2} \beta_{p p}=\beta_{k l}^{2} \beta_{l p}^{2} \quad \text { for } 1 \leq k<l<p \leq m .
$$

Therefore, the generators $\alpha$ and $\beta_{k l}(1 \leq k \leq l \leq m)$ are algebraically dependent.

Let $(y, v)$ be a coordinate in $\mathcal{P}_{1, m}$ with $y>0$ and $v={ }^{t}\left(v_{1}, v_{2}, \ldots, v_{m}\right) \in$ $\mathbb{R}^{(m, 1)}$. Then using Formula (3.10), we can show that

$$
\Theta_{1, m}(\alpha)=2 y \frac{\partial}{\partial y} \quad \text { and } \quad \Theta_{1, m}\left(\beta_{k l}\right)=y \frac{\partial^{2}}{\partial v_{k} \partial v_{l}}, \quad 1 \leq k \leq l \leq m .
$$

We see that $\Theta_{1, m}(\alpha)$ and $\Theta_{1, m}\left(\beta_{k l}\right)(1 \leq k \leq l \leq m)$ generate the algebra $\mathbb{D}\left(\mathcal{P}_{1, m}\right)$. Although $\Theta_{1, m}\left(\beta_{k l}\right)(1 \leq k \leq l \leq m)$ commute with each other, $\Theta_{1, m}(\alpha)$ does not commute with any $\Theta_{1, m}\left(\beta_{k l}\right)$. Indeed, we have the noncommutation relation

$$
\Theta_{1, m}(\alpha) \Theta_{1, m}\left(\beta_{k l}\right)-\Theta_{1, m}\left(\beta_{k l}\right) \Theta_{1, m}(\alpha)=2 \Theta_{1, m}\left(\beta_{k l}\right) .
$$

Hence the algebra $\mathbb{D}\left(\mathcal{P}_{1, m}\right)$ is not commutative. It is easily seen that the action of $K$ on $\operatorname{Pol}\left(\mathfrak{p}_{\star}\right)$ is not multiplicity-free.

\section{The case when $n=2$}

In this section, we deal with the case when $n=2, m=1$ and the case when $n=m=2$.

\subsection{The case when $n=2$ and $m=1$}

In this case,

$G L_{2,1}=G L(2, \mathbb{R}) \ltimes \mathbb{R}^{(1,2)}, \quad K=O(2)$ and $G L_{2,1} / K=\mathcal{P}_{2} \times \mathbb{R}^{(1,2)}=\mathcal{P}_{2,1}$.

We see easily that

$$
\mathfrak{p}_{\star}=\left\{(X, Z) \mid X={ }^{t} X \in \mathbb{R}^{(2,2)}, \quad Z \in \mathbb{R}^{(1,2)}\right\} .
$$

Put

$$
e_{1}=\left(\left(\begin{array}{ll}
1 & 0 \\
0 & 0
\end{array}\right), 0\right), \quad e_{2}=\left(\left(\begin{array}{ll}
0 & 0 \\
0 & 1
\end{array}\right), 0\right), \quad e_{3}=\left(\left(\begin{array}{ll}
0 & 1 \\
1 & 0
\end{array}\right), 0\right)
$$

and

$$
f_{1}=(0,(1,0)), \quad f_{2}=(0,(0,1)) .
$$

Then $\left\{e_{1}, e_{2}, e_{3}, f_{1}, f_{2}\right\}$ forms a basis for $\mathfrak{p}_{\star}$. For variables $(X, Z) \in \mathfrak{p}_{\star}$, write

$$
X=\left(\begin{array}{cc}
x_{1} & \frac{1}{2} x_{3} \\
\frac{1}{2} x_{3} & x_{2}
\end{array}\right) \quad \text { and } \quad Z=\left(z_{1}, z_{2}\right) .
$$

The following polynomials

$$
\begin{gathered}
\alpha_{1}(X, Z)=\operatorname{tr}(X)=x_{1}+x_{2}, \quad \alpha_{2}(X, Z)=\operatorname{tr}\left(X^{2}\right)=x_{1}^{2}+x_{2}^{2}+\frac{1}{2} x_{3}^{2}, \\
\xi(X, Z)=Z^{t} Z=z_{1}^{2}+z_{2}^{2}
\end{gathered}
$$


and

$$
\varphi(X, Z)=Z X^{t} Z=x_{1} z_{1}^{2}+x_{2} z_{2}^{2}+x_{3} z_{1} z_{2}
$$

generate the algebra $\operatorname{Pol}\left(\mathfrak{p}_{\star}\right)^{K}$. We can show that the invariants $\alpha_{1}, \alpha_{2}, \xi$ and $\varphi$ are algebraically independent. We omit the detail.

Now we compute the $G L_{2,1}$-invariant differential operators $D_{1}, D_{2}, \Psi, \Delta$ on $\mathcal{P}_{2,1}$ corresponding to the $K$-invariants $\alpha_{1}, \alpha_{2}, \xi, \varphi$, respectively, under a canonical linear bijection

$$
\Theta_{2,1}: \operatorname{Pol}\left(\mathfrak{p}_{\star}\right)^{K} \longrightarrow \mathbb{D}\left(\mathcal{P}_{2,1}\right)
$$

For real variables $t=\left(t_{1}, t_{2}, t_{3}\right)$ and $s=\left(s_{1}, s_{2}\right)$, we have

$$
\begin{gathered}
\exp \left(t_{1} e_{1}+t_{2} e_{2}+t_{3} e_{3}+s_{1} f_{1}+s_{2} f_{2}\right) \\
=\left(\left(\begin{array}{ll}
a_{1}(t, s) & a_{3}(t, s) \\
a_{3}(t, s) & a_{2}(t, s)
\end{array}\right),\left(b_{1}(t, s), b_{2}(t, s)\right)\right),
\end{gathered}
$$

where

$$
\begin{aligned}
& a_{1}(t, s)=1+t_{1}+\frac{1}{2 !}\left(t_{1}^{2}+t_{3}^{2}\right)+\frac{1}{3 !}\left(t_{1}^{3}+2 t_{1} t_{3}^{2}+t_{2} t_{3}^{2}\right)+\cdots, \\
& a_{2}(t, s)=1+t_{2}+\frac{1}{2 !}\left(t_{2}^{2}+t_{3}^{2}\right)+\frac{1}{3 !}\left(t_{1} t_{3}^{2}+2 t_{2} t_{3}^{2}+t_{2}^{3}\right)+\cdots, \\
& a_{3}(t, s)=t_{3}+\frac{1}{2 !}\left(t_{1}+t_{2}\right) t_{3}+\frac{1}{3 !}\left(t_{1} t_{2}+t_{1}^{2}+t_{2}^{2}+t_{3}^{2}\right) t_{3}+\cdots, \\
& b_{1}(t, s)=s_{1}-\frac{1}{2 !}\left(s_{1} t_{1}+s_{2} t_{3}\right)+\frac{1}{3 !}\left\{s_{1}\left(t_{1}^{2}+t_{3}^{2}\right)+s_{2}\left(t_{1} t_{3}+t_{2} t_{3}\right)\right\}-\cdots, \\
& b_{2}(t, s)=s_{2}-\frac{1}{2 !}\left(s_{1} t_{3}+s_{2} t_{2}\right)+\frac{1}{3 !}\left\{s_{1}\left(t_{1}+t_{2}\right) t_{3}+s_{2}\left(t_{2}^{2}+t_{3}^{2}\right)\right\}-\cdots .
\end{aligned}
$$

For brevity, we write $a_{i}, b_{k}$ for $a_{i}(t, s), b_{k}(t, s)(i=1,2,3, k=1,2)$, respectively. We now fix an element $(g, c) \in G L_{2,1}$ and write

$$
g=\left(\begin{array}{cc}
g_{1} & g_{12} \\
g_{21} & g_{2}
\end{array}\right) \text { and } c=\left(c_{1}, c_{2}\right)
$$

Put

$$
(Y(t, s), V(t, s))=\left((g, c) \cdot \exp \left(\sum_{i=1}^{3} t_{i} e_{i}+\sum_{k=1}^{2} s_{k} f_{k}\right)\right) \cdot\left(I_{2}, 0\right)
$$

with

$$
Y(t, s)=\left(\begin{array}{ll}
y_{1}(t, s) & y_{3}(t, s) \\
y_{3}(t, s) & y_{2}(t, s)
\end{array}\right) \quad \text { and } \quad V(t, s)=\left(v_{1}(t, s), v_{2}(t, s)\right) .
$$

By an easy computation, we obtain

$$
\begin{aligned}
& y_{1}=\left(g_{1} a_{1}+g_{12} a_{3}\right)^{2}+\left(g_{1} a_{3}+g_{12} a_{2}\right)^{2}, \\
& y_{2}=\left(g_{21} a_{1}+g_{2} a_{3}\right)^{2}+\left(g_{21} a_{3}+g_{2} a_{2}\right)^{2}, \\
& y_{3}=\left(g_{1} a_{1}+g_{12} a_{3}\right)\left(g_{21} a_{1}+g_{2} a_{3}\right)+\left(g_{1} a_{3}+g_{12} a_{2}\right)\left(g_{21} a_{3}+g_{2} a_{2}\right),
\end{aligned}
$$




$$
\begin{aligned}
& v_{1}=\left(c_{1}+b_{1} a_{1}+b_{2} a_{3}\right) g_{1}+\left(c_{2}+b_{1} a_{3}+b_{2} a_{2}\right) g_{12}, \\
& v_{2}=\left(c_{1}+b_{1} a_{1}+b_{2} a_{3}\right) g_{21}+\left(c_{2}+b_{1} a_{3}+b_{2} a_{2}\right) g_{2} .
\end{aligned}
$$

Using the chain rule, we can easily compute the $G L_{2,1}$-invariant differential operators $D_{1}=\Theta_{2,1}\left(\alpha_{1}\right), D_{2}=\Theta_{2,1}\left(\alpha_{2}\right), \Psi=\Theta_{2,1}(\xi)$ and $\Delta=\Theta_{2,1}(\varphi)$. They are given by

$$
\begin{aligned}
D_{1}= & 2 \operatorname{tr}\left(Y \frac{\partial}{\partial Y}\right)=2\left(y_{1} \frac{\partial}{\partial y_{1}}+y_{2} \frac{\partial}{\partial y_{2}}+y_{3} \frac{\partial}{\partial y_{3}}\right) \\
D_{2}= & \operatorname{tr}\left(\left(2 Y \frac{\partial}{\partial Y}\right)^{2}\right) \\
= & 3 D_{1}+8\left(y_{3}^{2} \frac{\partial^{2}}{\partial y_{1} \partial y_{2}}+y_{1} y_{3} \frac{\partial^{2}}{\partial y_{1} \partial y_{3}}+y_{2} y_{3} \frac{\partial^{2}}{\partial y_{2} \partial y_{3}}\right) \\
& +4\left\{y_{1}^{2} \frac{\partial^{2}}{\partial y_{1}^{2}}+y_{2}^{2} \frac{\partial^{2}}{\partial y_{2}^{2}}+\frac{1}{2}\left(y_{1} y_{2}+y_{3}^{2}\right) \frac{\partial^{2}}{\partial y_{3}^{2}}\right\} \\
\Psi= & \operatorname{tr}\left(Y^{t}\left(\frac{\partial}{\partial V}\right)\left(\frac{\partial}{\partial V}\right)\right) \\
= & y_{1} \frac{\partial^{2}}{\partial v_{1}^{2}}+2 y_{3} \frac{\partial^{2}}{\partial v_{1} \partial v_{2}}+y_{2} \frac{\partial^{2}}{\partial v_{2}^{2}}
\end{aligned}
$$

and

$$
\begin{aligned}
\Delta= & \frac{\partial}{\partial V}\left(2 Y \frac{\partial}{\partial Y}\right) Y^{t}\left(\frac{\partial}{\partial V}\right) \\
= & 2\left(y_{1}^{2} \frac{\partial^{3}}{\partial y_{1} \partial v_{1}^{2}}+2 y_{1} y_{3} \frac{\partial^{3}}{\partial y_{1} \partial v_{1} \partial v_{2}}+y_{3}^{2} \frac{\partial^{3}}{\partial y_{1} \partial v_{2}^{2}}\right) \\
& +2\left(y_{3}^{2} \frac{\partial^{3}}{\partial y_{2} \partial v_{1}^{2}}+2 y_{2} y_{3} \frac{\partial^{3}}{\partial y_{2} \partial v_{1} \partial v_{2}}+y_{2}^{2} \frac{\partial^{3}}{\partial y_{2} \partial v_{2}^{2}}\right) \\
& +2\left\{y_{1} y_{3} \frac{\partial^{3}}{\partial y_{3} \partial v_{1}^{2}}+\left(y_{1} y_{2}+y_{3}^{2}\right) \frac{\partial^{3}}{\partial y_{3} \partial v_{1} \partial v_{2}}+y_{2} y_{3} \frac{\partial^{3}}{\partial y_{3} \partial v_{2}^{2}}\right\} \\
& +3\left(y_{1} \frac{\partial^{2}}{\partial v_{1}^{2}}+2 y_{3} \frac{\partial^{2}}{\partial v_{1} \partial v_{2}}+y_{2} \frac{\partial^{2}}{\partial v_{2}^{2}}\right) .
\end{aligned}
$$

Clearly, $D_{1}$ commutes with $D_{2}$ but $\Psi$ does not commute with $D_{1}$ nor with $D_{2}$. Indeed, we have the following noncommutation relations

$$
\left[D_{1}, \Psi\right]=D_{1} \Psi-\Psi D_{1}=2 \Psi
$$

and

$$
\begin{aligned}
{\left[D_{2}, \Psi\right] } & =D_{2} \Psi-\Psi D_{2} \\
& =2\left(2 D_{1}-1\right) \Psi-8 \operatorname{det}(Y) \cdot \operatorname{det}\left(\frac{\partial}{\partial Y}+{ }^{t}\left(\frac{\partial}{\partial V}\right) \frac{\partial}{\partial V}\right)
\end{aligned}
$$




$$
+8 \operatorname{det}(Y) \cdot \operatorname{det}\left(\frac{\partial}{\partial Y}\right)-4\left(y_{1} y_{2}+y_{3}^{2}\right) \frac{\partial^{3}}{\partial y_{3} \partial v_{1} \partial v_{2}} .
$$

Hence the algebra $\mathbb{D}\left(\mathcal{P}_{2,1}\right)$ is not commutative.

\subsection{The case when $n=2$ and $m=2$}

In this case,

$$
G L_{2,2}=G L(2, \mathbb{R}) \ltimes \mathbb{R}^{(2,2)}, \quad K=O(2) \text { and } G L_{2,2} / K=\mathcal{P}_{2} \times \mathbb{R}^{(2,2)}=\mathcal{P}_{2,2} .
$$

We see easily that

$$
\mathfrak{p}_{\star}=\left\{(X, Z) \mid X={ }^{t} X \in \mathbb{R}^{(2,2)}, \quad Z \in \mathbb{R}^{(2,2)}\right\} .
$$

Let $\mathrm{O}_{2}$ be the $2 \times 2$ zero matrix. Put

$$
e_{1}=\left(\left(\begin{array}{cc}
1 & 0 \\
0 & 0
\end{array}\right), O_{2}\right), \quad e_{2}=\left(\left(\begin{array}{cc}
0 & 0 \\
0 & 1
\end{array}\right), O_{2}\right), \quad e_{3}=\left(\left(\begin{array}{cc}
0 & \frac{1}{2} \\
\frac{1}{2} & 0
\end{array}\right), O_{2}\right)
$$

and

$$
\begin{aligned}
& f_{1}=\left(O_{2},\left(\begin{array}{ll}
1 & 0 \\
0 & 0
\end{array}\right)\right), f_{2}=\left(O_{2},\left(\begin{array}{ll}
0 & 1 \\
0 & 0
\end{array}\right)\right), \\
& f_{3}=\left(O_{2},\left(\begin{array}{ll}
0 & 0 \\
1 & 0
\end{array}\right)\right), f_{4}=\left(O_{2},\left(\begin{array}{ll}
0 & 0 \\
0 & 1
\end{array}\right)\right) .
\end{aligned}
$$

Then $\left\{e_{1}, e_{2}, e_{3}, f_{1}, f_{2}, f_{3}, f_{4}\right\}$ forms a basis for $\mathfrak{p}_{\star}$. For variables $(X, Z) \in \mathfrak{p}_{\star}$, write

$$
X=\left(\begin{array}{cc}
x_{1} & \frac{1}{2} x_{3} \\
\frac{1}{2} x_{3} & x_{2}
\end{array}\right) \quad \text { and } \quad Z=\left(\begin{array}{ll}
z_{11} & z_{12} \\
z_{21} & z_{22}
\end{array}\right) .
$$

From Theorem 3.3, the algebra $\operatorname{Pol}\left(\mathfrak{p}_{\star}\right)^{K}$ is generated by the following polynomials

$$
\begin{aligned}
\alpha_{1}(X, Z) & =\operatorname{tr}(X)=x_{1}+x_{2}, \\
\alpha_{2}(X, Z) & =\operatorname{tr}\left(X^{2}\right)=x_{1}^{2}+x_{2}^{2}+\frac{1}{2} x_{3}^{2}, \\
\beta_{11}^{(0)}(X, Z) & =\left(Z^{t} Z\right)_{11}=z_{11}^{2}+z_{12}^{2}, \\
\beta_{12}^{(0)}(X, Z) & =\left(Z^{t} Z\right)_{12}=z_{11} z_{21}+z_{12} z_{22}, \\
\beta_{22}^{(0)}(X, Z) & =\left(Z^{t} Z\right)_{22}=z_{21}^{2}+z_{22}^{2}, \\
\beta_{11}^{(1)}(X, Z) & =\left(Z X^{t} Z\right)_{11}=x_{1} z_{11}^{2}+x_{2} z_{12}^{2}+x_{3} z_{11} z_{12}, \\
\beta_{12}^{(1)}(X, Z) & =\left(Z X^{t} Z\right)_{12}=x_{1} z_{11} z_{21}+x_{2} z_{12} z_{22}+\frac{1}{2} x_{3}\left(z_{11} z_{22}+z_{12} z_{21}\right), \\
\beta_{22}^{(1)}(X, Z) & =\left(Z X^{t} Z\right)_{22}=x_{1} z_{21}^{2}+x_{2} z_{22}^{2}+x_{3} z_{21} z_{22} .
\end{aligned}
$$


Set

$$
\Delta_{a b}:=\operatorname{det}\left(\begin{array}{cc}
\beta_{11}^{(a)} & \beta_{12}^{(b)} \\
\beta_{12}^{(a)} & \beta_{22}^{(b)}
\end{array}\right) \quad \text { for } a, b=0,1 .
$$

By a direct computation, we can show that the following equation

$$
\alpha_{1} \Delta_{00}-\Delta_{01}-\Delta_{10}=0
$$

holds.

We take a coordinate $(Y, V)$ in $\mathcal{P}_{2,2}$, that is,

$$
Y=\left(\begin{array}{ll}
y_{1} & y_{3} \\
y_{3} & y_{2}
\end{array}\right) \quad \text { and } \quad V=\left(\begin{array}{ll}
v_{11} & v_{12} \\
v_{21} & v_{22}
\end{array}\right)
$$

Put

$$
\frac{\partial}{\partial Y}=\left(\begin{array}{cc}
\frac{\partial}{\partial y_{1}} & \frac{1}{2} \frac{\partial}{\partial y_{3}} \\
\frac{1}{2} \frac{\partial}{\partial y_{3}} & \frac{\partial}{\partial y_{2}}
\end{array}\right) \quad \text { and } \quad \frac{\partial}{\partial V}=\left(\begin{array}{ll}
\frac{\partial}{\partial v_{11}} & \frac{\partial}{\partial v_{12}} \\
\frac{\partial}{\partial v_{21}} & \frac{\partial}{\partial v_{22}}
\end{array}\right)
$$

Consider the following differential operators

$$
D_{i}:=\operatorname{tr}\left(\left(2 Y \frac{\partial}{\partial Y}\right)^{i}\right), \quad i=1,2
$$

and

$$
\Omega_{p q}^{(k)}=\left\{\frac{\partial}{\partial V}\left(2 Y \frac{\partial}{\partial Y}\right)^{k} Y^{t}\left(\frac{\partial}{\partial V}\right)\right\}_{p q}, \quad k=0,1,1 \leq p \leq q \leq 2 .
$$

Note that $D_{1}, D_{2}, \Omega_{11}^{(0)}, \ldots, \Omega_{22}^{(1)}$ are $G L_{2,2}$-invariant. For brevity, we put

$$
\partial_{i j}=\frac{\partial}{\partial v_{i j}}, \quad i, j=1,2 \text {. }
$$

It is easily seen that

$$
\begin{aligned}
D_{1}= & \operatorname{tr}\left(2 Y \frac{\partial}{\partial Y}\right)=2 \sum_{i=1}^{3} y_{i} \frac{\partial}{\partial y_{i}} \\
D_{2}= & 3 D_{1}+8\left(y_{3}^{2} \frac{\partial^{2}}{\partial y_{1} \partial y_{2}}+y_{1} y_{3} \frac{\partial^{2}}{\partial y_{1} \partial y_{3}}+y_{2} y_{3} \frac{\partial^{2}}{\partial y_{2} \partial y_{3}}\right) \\
& +4\left\{y_{1}^{2} \frac{\partial^{2}}{\partial y_{1}^{2}}+y_{2}^{2} \frac{\partial^{2}}{\partial y_{2}^{2}}+\frac{1}{2}\left(y_{1} y_{2}+y_{3}^{2}\right) \frac{\partial^{2}}{\partial y_{3}^{2}}\right\} \\
\Omega_{11}^{(0)}= & y_{1} \partial_{11}^{2}+y_{2} \partial_{12}^{2}+2 y_{3} \partial_{11} \partial_{12} \\
\Omega_{12}^{(0)}= & y_{1} \partial_{11} \partial_{21}+y_{2} \partial_{12} \partial_{22}+y_{3}\left(\partial_{11} \partial_{22}+\partial_{12} \partial_{21}\right) \\
\Omega_{22}^{(0)}= & y_{1} \partial_{21}^{2}+y_{2} \partial_{22}^{2}+2 y_{3} \partial_{21} \partial_{22} .
\end{aligned}
$$

Then by a direct computation, we have the following relations

$$
\left[D_{1}, D_{2}\right]=0 \text {, }
$$




$$
\begin{gathered}
{\left[\Omega_{k l}^{(0)}, \Omega_{p q}^{(0)}\right]=0, \quad 1 \leq k \leq l \leq 2,1 \leq p \leq q \leq 2,} \\
{\left[D_{1}, \Omega_{11}^{(0)}\right]=2 \Omega_{11}^{(0)}, \quad\left[D_{1}, \Omega_{12}^{(0)}\right]=2 \Omega_{12}^{(0)}, \quad\left[D_{1}, \Omega_{22}^{(0)}\right]=2 \Omega_{22}^{(0)} .}
\end{gathered}
$$

Therefore, $\mathbb{D}\left(\mathcal{P}_{2,2}\right)$ is not commutative.

\section{The case when $n=3$}

\subsection{The case when $n=3$ and $m=1$}

In this case,

$G L_{3,1}=G L(3, \mathbb{R}) \ltimes \mathbb{R}^{(1,3)}, \quad K=O(3)$ and $G L_{3,1} / K=\mathcal{P}_{3} \times \mathbb{R}^{(1,3)}=\mathcal{P}_{3,1}$.

We see easily that

$$
\mathfrak{p}_{\star}=\left\{(X, Z) \mid X={ }^{t} X \in \mathbb{R}^{(3,3)}, \quad Z \in \mathbb{R}^{(1,3)}\right\} .
$$

Put

$$
\begin{aligned}
& E_{1}=\left(\begin{array}{lll}
1 & 0 & 0 \\
0 & 0 & 0 \\
0 & 0 & 0
\end{array}\right), \quad E_{2}=\left(\begin{array}{lll}
0 & 0 & 0 \\
0 & 1 & 0 \\
0 & 0 & 0
\end{array}\right), \quad E_{3}=\left(\begin{array}{lll}
0 & 0 & 0 \\
0 & 0 & 0 \\
0 & 0 & 1
\end{array}\right), \\
& E_{4}=\left(\begin{array}{ccc}
0 & \frac{1}{2} & 0 \\
\frac{1}{2} & 0 & 0 \\
0 & 0 & 0
\end{array}\right), \quad E_{5}=\left(\begin{array}{ccc}
0 & 0 & \frac{1}{2} \\
0 & 0 & 0 \\
\frac{1}{2} & 0 & 0
\end{array}\right), \quad E_{6}=\left(\begin{array}{ccc}
0 & 0 & 0 \\
0 & 0 & \frac{1}{2} \\
0 & \frac{1}{2} & 0
\end{array}\right) \text {. }
\end{aligned}
$$

Let $O_{3}$ be the $3 \times 3$ zero matrix and let $O_{1,3}=(0,0,0) \in \mathbb{R}^{(1,3)}$. Put

$$
\begin{aligned}
& e_{i}=\left(E_{i}, O_{1,3}\right), \quad 1 \leq i \leq 6, \\
& f_{1}=\left(O_{3},(1,0,0)\right), \quad f_{2}=\left(O_{3},(0,1,0)\right), \quad f_{3}=\left(O_{3},(0,0,1)\right) .
\end{aligned}
$$

Then $\left\{e_{i}, f_{j} \mid 1 \leq i \leq 6,1 \leq j \leq 3\right\}$ forms a basis for $\mathfrak{p}_{\star}$. Using this basis, we write for variables $(X, Z) \in \mathfrak{p}_{\star}$,

$$
X=\left(\begin{array}{ccc}
x_{1} & \frac{1}{2} x_{4} & \frac{1}{2} x_{5} \\
\frac{1}{2} x_{4} & x_{2} & \frac{1}{2} x_{6} \\
\frac{1}{2} x_{5} & \frac{1}{2} x_{6} & x_{3}
\end{array}\right) \quad \text { and } \quad Z=\left(z_{1}, z_{2}, z_{3}\right) .
$$

From Theorem 3.3, the algebra $\operatorname{Pol}\left(\mathfrak{p}_{\star}\right)^{K}$ is generated by the following polynomials

$$
\begin{aligned}
\alpha_{1}(X, Z)= & x_{1}+x_{2}+x_{3}, \\
\alpha_{2}(X, Z)= & x_{1}^{2}+x_{2}^{2}+x_{3}^{2}+\frac{1}{2}\left(x_{4}^{2}+x_{5}^{2}+x_{6}^{2}\right), \\
\alpha_{3}(X, Z)= & x_{1}^{3}+x_{2}^{3}+x_{3}^{3}+\frac{3}{4}\left\{\left(x_{1}+x_{2}\right) x_{4}^{2}+\left(x_{1}+x_{3}\right) x_{5}^{2}+\left(x_{2}+x_{3}\right) x_{6}^{2}\right\} \\
& +\frac{3}{4} x_{4} x_{5} x_{6}, \\
\beta_{0}(X, Z)= & z_{1}^{2}+z_{2}^{2}+z_{3}^{2},
\end{aligned}
$$




$$
\begin{aligned}
\beta_{1}(X, Z)= & x_{1} z_{1}^{2}+x_{2} z_{2}^{2}+x_{3} z_{3}^{2}+x_{4} z_{1} z_{2}+x_{5} z_{1} z_{3}+x_{6} z_{2} z_{3}, \\
\beta_{2}(X, Z)= & x_{1}^{2} z_{1}^{2}+x_{2}^{2} z_{2}^{2}+\frac{1}{4}\left\{\left(x_{4}^{2}+x_{5}^{2}\right) z_{1}^{2}+\left(x_{4}^{2}+x_{6}^{2}\right) z_{2}^{2}+\left(x_{5}^{2}+x_{6}^{2}\right) z_{3}^{2}\right\} \\
& +\left(x_{1} x_{4}+x_{2} x_{4}+\frac{1}{2} x_{5} x_{6}\right) z_{1} z_{2}+\left(x_{1} x_{5}+x_{3} x_{5}+\frac{1}{2} x_{4} x_{6}\right) z_{1} z_{3} \\
& +\left(x_{2} x_{6}+x_{3} x_{6}+\frac{1}{2} x_{4} x_{5}\right) z_{2} z_{3} .
\end{aligned}
$$

We take a coordinate $(Y, V)$ in $\mathcal{P}_{3,1}$, that is,

$$
Y=\left(\begin{array}{lll}
y_{1} & y_{4} & y_{5} \\
y_{4} & y_{2} & y_{6} \\
y_{5} & y_{6} & y_{3}
\end{array}\right) \quad \text { and } \quad V=\left(v_{1}, v_{2}, v_{3}\right)
$$

Put

$$
\frac{\partial}{\partial Y}=\left(\begin{array}{ccc}
\frac{\partial}{\partial y_{1}} & \frac{1}{2} \frac{\partial}{\partial y_{4}} & \frac{1}{2} \frac{\partial}{\partial y_{5}} \\
\frac{1}{2} \frac{\partial}{\partial y_{4}} & \frac{\partial}{\partial y_{2}} & \frac{1}{2} \frac{\partial}{\partial y_{6}} \\
\frac{1}{2} \frac{\partial}{\partial y_{5}} & \frac{1}{2} \frac{\frac{1}{\partial y_{6}}}{\partial y_{3}} & \frac{\partial}{\partial y_{3}}
\end{array}\right) \quad \text { and } \quad \frac{\partial}{\partial V}=\left(\frac{\partial}{\partial v_{1}}, \frac{\partial}{\partial v_{2}}, \frac{\partial}{\partial v_{3}}\right)
$$

Consider the following differential operators

$$
D_{i}:=\operatorname{tr}\left(\left(2 Y \frac{\partial}{\partial Y}\right)^{i}\right), \quad i=1,2,3
$$

and

$$
\Omega_{k}=\frac{\partial}{\partial V}\left(2 Y \frac{\partial}{\partial Y}\right)^{k} Y^{t}\left(\frac{\partial}{\partial V}\right), \quad k=0,1,2 .
$$

Note that $D_{1}, D_{2}, D_{3}, \Omega_{0}, \Omega_{1}$ and $\Omega_{2}$ are $G L_{2,2}$-invariant. It is easily seen that

$$
\begin{aligned}
D_{1}= & \operatorname{tr}\left(2 Y \frac{\partial}{\partial Y}\right)=2 \sum_{i=1}^{6} y_{i} \frac{\partial}{\partial y_{i}}, \\
\Omega_{0}= & y_{1} \frac{\partial^{2}}{\partial v_{1}^{2}}+y_{2} \frac{\partial^{2}}{\partial v_{2}^{2}}+y_{3} \frac{\partial^{2}}{\partial v_{3}^{2}} \\
& +2 y_{4} \frac{\partial^{2}}{\partial v_{1} \partial v_{2}}+2 y_{5} \frac{\partial^{2}}{\partial v_{1} \partial v_{3}}+2 y_{6} \frac{\partial^{2}}{\partial v_{2} \partial v_{3}} .
\end{aligned}
$$

Then we have the following relations

$$
\left[D_{i}, D_{j}\right]=0 \quad \text { for all } i, j=1,2,3
$$

and

$$
\left[D_{1}, \Omega_{0}\right]=2 \Omega_{0} .
$$

Therefore, $\mathbb{D}\left(\mathcal{P}_{3,1}\right)$ is not commutative.

\subsection{The case when $n=3$ and $m=2$}


In this case,

$G L_{3,2}=G L(3, \mathbb{R}) \ltimes \mathbb{R}^{(2,3)}, \quad K=O(3)$ and $G L_{3,2} / K=\mathcal{P}_{3} \times \mathbb{R}^{(2,3)}=\mathcal{P}_{3,2}$.

We see easily that

$$
\mathfrak{p}_{\star}=\left\{(X, Z) \mid X={ }^{t} X \in \mathbb{R}^{(3,3)}, \quad Z \in \mathbb{R}^{(2,3)}\right\} .
$$

Put

$$
\begin{aligned}
& E_{1}=\left(\begin{array}{lll}
1 & 0 & 0 \\
0 & 0 & 0 \\
0 & 0 & 0
\end{array}\right), \quad E_{2}=\left(\begin{array}{lll}
0 & 0 & 0 \\
0 & 1 & 0 \\
0 & 0 & 0
\end{array}\right), \quad E_{3}=\left(\begin{array}{lll}
0 & 0 & 0 \\
0 & 0 & 0 \\
0 & 0 & 1
\end{array}\right), \\
& E_{4}=\left(\begin{array}{ccc}
0 & \frac{1}{2} & 0 \\
\frac{1}{2} & 0 & 0 \\
0 & 0 & 0
\end{array}\right), \quad E_{5}=\left(\begin{array}{ccc}
0 & 0 & \frac{1}{2} \\
0 & 0 & 0 \\
\frac{1}{2} & 0 & 0
\end{array}\right), \quad E_{6}=\left(\begin{array}{ccc}
0 & 0 & 0 \\
0 & 0 & \frac{1}{2} \\
0 & \frac{1}{2} & 0
\end{array}\right) \text {. }
\end{aligned}
$$

and

$$
\begin{array}{ll}
F_{1}=\left(\begin{array}{lll}
1 & 0 & 0 \\
0 & 0 & 0
\end{array}\right), & F_{2}=\left(\begin{array}{lll}
0 & 1 & 0 \\
0 & 0 & 0
\end{array}\right), \quad F_{3}=\left(\begin{array}{lll}
0 & 0 & 1 \\
0 & 0 & 0
\end{array}\right), \\
F_{4}=\left(\begin{array}{lll}
0 & 0 & 0 \\
1 & 0 & 0
\end{array}\right), & F_{5}=\left(\begin{array}{lll}
0 & 0 & 0 \\
0 & 1 & 0
\end{array}\right),
\end{array}
$$

Let $O_{3}$ be the $3 \times 3$ zero matrix and let

$$
O_{2,3}=\left(\begin{array}{ccc}
0 & 0 & 0 \\
0 & 0 & 0
\end{array}\right) \in \mathbb{R}^{(2,3)}
$$

Put

$$
e_{i}=\left(E_{i}, O_{2,3}\right), \quad f_{j}=\left(O_{3}, F_{j}\right) \quad 1 \leq i, j \leq 6 .
$$

Then $\left\{e_{i}, f_{j} \mid 1 \leq i, j \leq 6\right\}$ forms a basis for $\mathfrak{p}_{\star}$. Using this basis, we write for variables $(X, Z) \in \mathfrak{p}_{\star}$,

$$
X=\left(\begin{array}{ccc}
x_{1} & \frac{1}{2} x_{4} & \frac{1}{2} x_{5} \\
\frac{1}{2} x_{4} & x_{2} & \frac{1}{2} x_{6} \\
\frac{1}{2} x_{5} & \frac{1}{2} x_{6} & x_{3}
\end{array}\right) \quad \text { and } \quad Z=\left(\begin{array}{ccc}
z_{11} & z_{12} & z_{13} \\
z_{21} & z_{22} & z_{23}
\end{array}\right)
$$

From Theorem 3.3, the algebra $\operatorname{Pol}\left(\mathfrak{p}_{\star}\right)^{K}$ is generated by the following polynomials

$$
\begin{aligned}
\alpha_{1}(X, Z)= & x_{1}+x_{2}+x_{3}, \\
\alpha_{2}(X, Z)= & x_{1}^{2}+x_{2}^{2}+x_{3}^{2}+\frac{1}{2}\left(x_{4}^{2}+x_{5}^{2}+x_{6}^{2}\right), \\
\alpha_{3}(X, Z)= & x_{1}^{3}+x_{2}^{3}+x_{3}^{3}+\frac{3}{4}\left\{\left(x_{1}+x_{2}\right) x_{4}^{2}+\left(x_{1}+x_{3}\right) x_{5}^{2}+\left(x_{2}+x_{3}\right) x_{6}^{2}\right\} \\
& +\frac{3}{4} x_{4} x_{5} x_{6}, \\
\beta_{11}^{(0)}(X, Z)= & z_{11}^{2}+z_{12}^{2}+z_{13}^{2}, \\
\beta_{12}^{(0)}(X, Z)= & z_{11} z_{21}+z_{12} z_{22}+z_{13} z_{23},
\end{aligned}
$$




$$
\begin{aligned}
& \beta_{22}^{(0)}(X, Z)=z_{21}^{2}+z_{22}^{2}+z_{23}^{2}, \\
& \beta_{11}^{(1)}(X, Z)=x_{1} z_{11}^{2}+x_{2} z_{12}^{2}+x_{3} z_{13}^{2}+x_{4} z_{11} z_{12}+x_{5} z_{11} z_{13}+x_{6} z_{12} z_{13}, \\
& \beta_{12}^{(1)}(X, Z)=x_{1} z_{11} z_{21}+x_{2} z_{12} z_{22}+x_{3} z_{13} z_{23}+\frac{1}{2} x_{4}\left(z_{11} z_{22}+z_{12} z_{21}\right) \\
& +\frac{1}{2} x_{5}\left(z_{11} z_{23}+z_{13} z_{21}\right)+\frac{1}{2} x_{6}\left(z_{12} z_{23}+z_{13} z_{22}\right), \\
& \beta_{22}^{(1)}(X, Z)=x_{1} z_{21}^{2}+x_{2} z_{22}^{2}+x_{3} z_{23}^{2}+x_{4} z_{21} z_{22}+x_{5} z_{21} z_{23}+x_{6} z_{22} z_{23} \text {, } \\
& \beta_{11}^{(2)}(X, Z)=x_{1}^{2} z_{11}^{2}+x_{2}^{2} z_{12}^{2}+x_{3}^{2} z_{13}^{2} \\
& +\frac{1}{4}\left\{x_{4}^{2}\left(z_{11}^{2}+z_{12}^{2}\right)+x_{5}^{2}\left(z_{11}^{2}+z_{13}^{2}\right)+x_{6}^{2}\left(z_{12}^{2}+z_{13}^{2}\right)\right\} \\
& +\left(x_{1}+x_{2}\right) x_{4} z_{11} z_{12}+\left(x_{1}+x_{3}\right) x_{5} z_{11} z_{13}+\left(x_{2}+x_{3}\right) x_{6} z_{12} z_{13} \\
& +\frac{1}{2}\left(x_{4} x_{5} z_{12} z_{13}+x_{4} x_{6} z_{11} z_{13}+x_{5} x_{6} z_{11} z_{12}\right) \text {, } \\
& \beta_{12}^{(2)}(X, Z)=x_{1}^{2} z_{11} z_{21}+x_{2}^{2} z_{12} z_{22}+x_{3}^{2} z_{13} z_{23} \\
& +\frac{1}{4}\left\{\left(x_{4}^{2}+x_{5}^{2}\right) z_{11} z_{21}+\left(x_{4}^{2}+x_{6}^{2}\right) z_{12} z_{22}+\left(x_{5}^{2}+x_{6}^{2}\right) z_{13} z_{23}\right\} \\
& +\frac{1}{2}\left(x_{1} x_{4}+x_{2} x_{4}+\frac{1}{2} x_{5} x_{6}\right)\left(z_{11} z_{22}+z_{12} z_{21}\right) \\
& +\frac{1}{2}\left(x_{1} x_{5}+x_{3} x_{5}+\frac{1}{2} x_{4} x_{6}\right)\left(z_{11} z_{23}+z_{13} z_{21}\right) \\
& +\frac{1}{2}\left(x_{2} x_{6}+x_{3} x_{6}+\frac{1}{2} x_{4} x_{5}\right)\left(z_{12} z_{23}+z_{13} z_{22}\right), \\
& \beta_{22}^{(2)}(X, Z)=x_{1}^{2} z_{21}^{2}+x_{2}^{2} z_{22}^{2}+x_{3}^{2} z_{23}^{2} \\
& +\frac{1}{4}\left\{x_{4}^{2}\left(z_{21}^{2}+z_{22}^{2}\right)+x_{5}^{2}\left(z_{21}^{2}+z_{23}^{2}\right)+x_{6}^{2}\left(z_{22}^{2}+z_{23}^{2}\right)\right\} \\
& +\left(x_{1}+x_{2}\right) x_{4} z_{21} z_{22}+\left(x_{1}+x_{3}\right) x_{5} z_{21} z_{23}+\left(x_{2}+x_{3}\right) x_{6} z_{22} z_{23} \\
& +\frac{1}{2}\left(x_{4} x_{5} z_{22} z_{23}+x_{4} x_{6} z_{21} z_{23}+x_{5} x_{6} z_{21} z_{22}\right) \text {. }
\end{aligned}
$$

Set

$$
\Delta_{a b}:=\operatorname{det}\left(\begin{array}{cc}
\beta_{11}^{(a)} & \beta_{12}^{(b)} \\
\beta_{12}^{(a)} & \beta_{22}^{(b)}
\end{array}\right) \quad \text { for } a, b=0,1,2 .
$$

By a direct computation, we can show that

$$
\left(\alpha_{1}^{2}-\alpha_{2}\right) \Delta_{00}-2 \alpha_{1}\left(\Delta_{01}+\Delta_{10}\right)+2\left(\Delta_{02}+\Delta_{11}+\Delta_{20}\right)=0 .
$$

We take a coordinate $(Y, V)$ in $\mathcal{P}_{3,2}$, that is,

$$
Y=\left(\begin{array}{lll}
y_{1} & y_{4} & y_{5} \\
y_{4} & y_{2} & y_{6} \\
y_{5} & y_{6} & y_{3}
\end{array}\right) \quad \text { and } \quad V=\left(\begin{array}{lll}
v_{11} & v_{12} & v_{13} \\
v_{21} & v_{22} & v_{23}
\end{array}\right)
$$


Put

$$
\frac{\partial}{\partial Y}=\left(\begin{array}{ccc}
\frac{\partial}{\partial y_{1}} & \frac{1}{2} \frac{\partial}{\partial y_{4}} & \frac{1}{2} \frac{\partial}{\partial y_{5}} \\
\frac{1}{2} \frac{\partial}{\partial y_{4}} & \frac{\partial}{\partial y_{2}} & \frac{1}{2} \frac{\partial}{\partial y_{6}} \\
\frac{1}{2} \frac{\partial}{\partial y_{5}} & \frac{1}{2} \frac{\partial}{\partial y_{6}} & \frac{\partial}{\partial y_{3}}
\end{array}\right) \quad \text { and } \quad\left(\begin{array}{lll}
\frac{\partial}{\partial v_{11}} & \frac{\partial}{\partial v_{12}} & \frac{\partial}{\partial v_{13}} \\
\frac{\partial}{\partial v_{21}} & \frac{\partial}{\partial v_{22}} & \frac{\partial}{\partial v_{23}}
\end{array}\right)
$$

Consider the following differential operators

$$
D_{i}:=\operatorname{tr}\left(\left(2 Y \frac{\partial}{\partial Y}\right)^{i}\right), \quad i=1,2,3
$$

and

$$
\Omega_{p q}^{(k)}=\left\{\frac{\partial}{\partial V}\left(2 Y \frac{\partial}{\partial Y}\right)^{k} Y^{t}\left(\frac{\partial}{\partial V}\right)\right\}_{p q}, \quad k=0,1,2,1 \leq p \leq q \leq 2 .
$$

Note that $D_{1}, D_{2}, D_{3}, \Omega_{11}^{(0)}, \ldots, \Omega_{22}^{(2)}$ are $G L_{3,2}$-invariant. For brevity, we put

$$
\partial_{i j}=\frac{\partial}{\partial v_{i j}}, \quad i=1,2, j=1,2,3 \text {. }
$$

It is easily seen that

$$
\begin{aligned}
D_{1}= & \operatorname{tr}\left(2 Y \frac{\partial}{\partial Y}\right)=2 \sum_{i=1}^{6} y_{i} \frac{\partial}{\partial y_{i}} \\
\Omega_{11}^{(0)}= & y_{1} \partial_{11}^{2}+y_{2} \partial_{12}^{2}+y_{3} \partial_{13}^{2}+2 y_{4} \partial_{11} \partial_{12}+2 y_{5} \partial_{11} \partial_{13}+2 y_{6} \partial_{12} \partial_{13}, \\
\Omega_{12}^{(0)}= & y_{1} \partial_{11} \partial_{21}+y_{2} \partial_{12} \partial_{22}+y_{3} \partial_{13} \partial_{23}+y_{4}\left(\partial_{11} \partial_{22}+\partial_{12} \partial_{21}\right) \\
& \quad+y_{5}\left(\partial_{11} \partial_{23}+\partial_{13} \partial_{21}\right)+y_{6}\left(\partial_{12} \partial_{23}+\partial_{13} \partial_{22}\right), \\
\Omega_{22}^{(0)}= & y_{1} \partial_{21}^{2}+y_{2} \partial_{22}^{2}+y_{3} \partial_{23}^{2}+2 y_{4} \partial_{21} \partial_{22}+2 y_{5} \partial_{21} \partial_{23}+2 y_{6} \partial_{22} \partial_{23} .
\end{aligned}
$$

Then we have the following relations

$$
\left[D_{i}, D_{j}\right]=0 \text { for all } i, j=1,2,3 \text {, }
$$

$$
\left[\Omega_{k l}^{(0)}, \Omega_{p q}^{(0)}\right]=0, \quad 1 \leq k \leq l \leq 2,1 \leq p \leq q \leq 2
$$

and

(6.6) $\quad\left[D_{1}, \Omega_{11}^{(0)}\right]=2 \Omega_{11}^{(0)}, \quad\left[D_{1}, \Omega_{12}^{(0)}\right]=2 \Omega_{12}^{(0)}, \quad\left[D_{1}, \Omega_{22}^{(0)}\right]=2 \Omega_{22}^{(0)}$.

Therefore, $\mathbb{D}\left(\mathcal{P}_{3,2}\right)$ is not commutative.

\section{The case when $n=4$}

\subsection{The case when $n=4$ and $m=1$}

In this case,

$G L_{4,1}=G L(4, \mathbb{R}) \ltimes \mathbb{R}^{(1,4)}, \quad K=O(4)$ and $G L_{4,1} / K=\mathcal{P}_{4} \times \mathbb{R}^{(1,4)}=\mathcal{P}_{4,1}$. 
We see easily that

$$
\mathfrak{p}_{\star}=\left\{(X, Z) \mid X={ }^{t} X \in \mathbb{R}^{(4,4)}, \quad Z \in \mathbb{R}^{(1,4)}\right\} .
$$

Put

$$
\begin{aligned}
& E_{1}=\left(\begin{array}{llll}
1 & 0 & 0 & 0 \\
0 & 0 & 0 & 0 \\
0 & 0 & 0 & 0 \\
0 & 0 & 0 & 0
\end{array}\right), E_{2}=\left(\begin{array}{cccc}
0 & 0 & 0 & 0 \\
0 & 1 & 0 & 0 \\
0 & 0 & 0 & 0 \\
0 & 0 & 0 & 0
\end{array}\right), E_{3}=\left(\begin{array}{llll}
0 & 0 & 0 & 0 \\
0 & 0 & 0 & 0 \\
0 & 0 & 1 & 0 \\
0 & 0 & 0 & 0
\end{array}\right), \\
& E_{4}=\left(\begin{array}{llll}
0 & 0 & 0 & 0 \\
0 & 0 & 0 & 0 \\
0 & 0 & 0 & 0 \\
0 & 0 & 0 & 1
\end{array}\right), E_{5}=\left(\begin{array}{cccc}
0 & \frac{1}{2} & 0 & 0 \\
\frac{1}{2} & 0 & 0 & 0 \\
0 & 0 & 0 & 0 \\
0 & 0 & 0 & 0
\end{array}\right), \quad E_{6}=\left(\begin{array}{cccc}
0 & 0 & \frac{1}{2} & 0 \\
0 & 0 & 0 & 0 \\
\frac{1}{2} & 0 & 0 & 0 \\
0 & 0 & 0 & 0
\end{array}\right), \\
& E_{7}=\left(\begin{array}{cccc}
0 & 0 & 0 & \frac{1}{2} \\
0 & 0 & 0 & 0 \\
0 & 0 & 0 & 0 \\
\frac{1}{2} & 0 & 0 & 0
\end{array}\right), E_{8}=\left(\begin{array}{cccc}
0 & 0 & 0 & 0 \\
0 & 0 & \frac{1}{2} & 0 \\
0 & \frac{1}{2} & 0 & 0 \\
0 & 0 & 0 & 0
\end{array}\right) \text {, } \\
& E_{9}=\left(\begin{array}{cccc}
0 & 0 & 0 & 0 \\
0 & 0 & 0 & \frac{1}{2} \\
0 & 0 & 0 & 0 \\
0 & \frac{1}{2} & 0 & 0
\end{array}\right), E_{10}=\left(\begin{array}{cccc}
0 & 0 & 0 & 0 \\
0 & 0 & 0 & 0 \\
0 & 0 & 0 & \frac{1}{2} \\
0 & 0 & \frac{1}{2} & 0
\end{array}\right) \text {. }
\end{aligned}
$$

Let $O_{4}$ be the $4 \times 4$ zero matrix and let $O_{1,4}=(0,0,0,0) \in \mathbb{R}^{(1,4)}$. Put

$$
\begin{aligned}
& e_{i}=\left(E_{i}, O_{1,4}\right), \quad 1 \leq i \leq 10, \\
& f_{1}=\left(O_{4},(1,0,0,0)\right), f_{2}=\left(O_{4},(0,1,0,0)\right), \\
& f_{3}=\left(O_{4},(0,0,1,0)\right), f_{4}=\left(O_{4},(0,0,0,1)\right) .
\end{aligned}
$$

Then $\left\{e_{i}, f_{j} \mid 1 \leq i \leq 10,1 \leq j \leq 4\right\}$ forms a basis for $\mathfrak{p}_{\star}$. Using this basis, we write for variables $(X, Z) \in \mathfrak{p}_{\star}$,

$$
X=\left(\begin{array}{cccc}
x_{1} & \frac{1}{2} x_{5} & \frac{1}{2} x_{6} & \frac{1}{2} x_{7} \\
\frac{1}{2} x_{5} & x_{2} & \frac{1}{2} x_{8} & \frac{1}{2} x_{9} \\
\frac{1}{2} x_{6} & \frac{1}{2} x_{8} & x_{3} & \frac{1}{2} x_{10} \\
\frac{1}{2} x_{7} & \frac{1}{2} x_{9} & \frac{1}{2} x_{10} & x_{4}
\end{array}\right) \text { and } Z=\left(z_{1}, z_{2}, z_{3}, z_{4}\right)
$$

Put

$$
\begin{aligned}
A & =x_{1}^{2}+\frac{1}{4} x_{5}^{2}+\frac{1}{4} x_{6}+\frac{1}{4} x_{7}^{2}, \\
B & =x_{2}^{2}+\frac{1}{4} x_{5}^{2}+\frac{1}{4} x_{8}+\frac{1}{4} x_{9}^{2}, \\
C & =x_{3}^{2}+\frac{1}{4} x_{6}^{2}+\frac{1}{4} x_{8}+\frac{1}{4} x_{10}^{2}, \\
D & =x_{4}^{2}+\frac{1}{4} x_{7}^{2}+\frac{1}{4} x_{9}+\frac{1}{4} x_{10}^{2},
\end{aligned}
$$




$$
\begin{aligned}
E & =\frac{1}{2}\left(x_{1}+x_{2}\right) x_{5}+\frac{1}{4}\left(x_{6} x_{8}+x_{7} x_{9}\right), \\
F & =\frac{1}{2}\left(x_{1}+x_{3}\right) x_{6}+\frac{1}{4}\left(x_{3} x_{6}+x_{5} x_{8}\right) \\
G & =\frac{1}{2}\left(x_{1}+x_{4}\right) x_{7}+\frac{1}{4}\left(x_{5} x_{9}+x_{6} x_{10}\right) \\
H & =\frac{1}{2}\left(x_{2}+x_{3}\right) x_{8}+\frac{1}{4}\left(x_{5} x_{6}+x_{9} x_{10}\right) \\
I & =\frac{1}{2}\left(x_{2}+x_{4}\right) x_{9}+\frac{1}{4}\left(x_{5} x_{7}+x_{8} x_{10}\right), \\
J & =\frac{1}{2}\left(x_{3}+x_{4}\right) x_{10}+\frac{1}{4}\left(x_{6} x_{10}+x_{6} x_{7}\right)
\end{aligned}
$$

From Theorem 3.3, the algebra $\operatorname{Pol}\left(\mathfrak{p}_{\star}\right)^{K}$ is generated by the following polynomials

$$
\begin{aligned}
& \alpha_{1}(X, Z)= x_{1}+x_{2}+x_{3}+x_{4}, \\
& \alpha_{2}(X, Z)=x_{1}^{2}+x_{2}^{2}+x_{3}^{2}+x_{4}^{2}+\frac{1}{2}\left(x_{5}^{2}+x_{6}^{2}+x_{7}^{2}+x_{8}^{2}+x_{9}^{2}+x_{10}^{2}\right), \\
& \alpha_{3}(X, Z)=x_{1}^{3}+x_{2}^{3}+x_{3}^{3}+x_{4}^{3} \\
&+\frac{3}{4} x_{1}\left(x_{5}^{2}+x_{6}^{2}+x_{7}^{2}\right)+\frac{3}{4} x_{2}\left(x_{5}^{2}+x_{8}^{2}+x_{9}^{2}\right) \\
&+\frac{3}{4} x_{3}\left(x_{6}^{2}+x_{8}^{2}+x_{10}^{2}\right)+\frac{3}{4} x_{4}\left(x_{7}^{2}+x_{9}^{2}+x_{10}^{2}\right) \\
&+\frac{3}{4}\left(x_{5} x_{6} x_{8}+x_{5} x_{7} x_{9}+x_{6} x_{7} x_{10}+x_{8} x_{9} x_{10}\right), \\
& \alpha_{4}(X, Z)=A^{2}+B^{2}+C^{2}+D^{2}+2\left(E^{2}+F^{2}+G^{2}+H^{2}+I^{2}+J^{2}\right), \\
& \beta_{0}(X, Z)= z_{1}^{2}+z_{2}^{2}+z_{3}^{2}+z_{4}^{2}, \\
& \beta_{1}(X, Z)= x_{1} z_{1}^{2}+x_{2} z_{2}^{2}+x_{3} z_{3}^{2}+x_{4} z_{4}^{2}, \\
&+x_{5} z_{1} z_{2}+x_{6} z_{1} z_{3}+x_{7} z_{1} z_{4}+x_{8} z_{2} z_{3}+x_{9} z_{2} z_{4}+x_{10} z_{3} z_{4}, \\
& \beta_{2}(X, Z)= A z_{1}^{2}+B z_{2}^{2}+C z_{3}^{2}+D z_{4}^{2}, \\
&+2\left(E z_{1} z_{2}+F z_{1} z_{3}+G z_{1} z_{4}+H z_{2} z_{3}+I z_{2} z_{4}+J z_{3} z_{4}\right), \\
& \beta_{3}(X, Z)= \frac{1}{2}\left(2 A x_{1}+E x_{5}+F x_{6}+G x_{7}\right) z_{1}^{2} \\
&+\frac{1}{2}\left(2 B x_{2}+E x_{5}+H x_{8}+I x_{9}\right) z_{2}^{2} \\
&+\frac{1}{2}\left(2 C x_{3}+F x_{6}+H x_{8}+J x_{10}\right) z_{3}^{2} \\
&+\frac{1}{2}\left(2 D x_{4}+G x_{7}+I x_{9}+J x_{10}\right) z_{4}^{2} \\
&+\frac{1}{2}\left\{2 E\left(x_{1}+x_{2}\right)+(A+B) x_{5}+H x_{6}+I x_{7}+F x_{8}+G x_{9}\right\} z_{1} z_{2}
\end{aligned}
$$




$$
\begin{aligned}
& +\frac{1}{2}\left\{2 F\left(x_{1}+x_{3}\right)+H x_{5}+(A+C) x_{6}+J x_{7}+E x_{8}+G x_{10}\right\} z_{1} z_{3} \\
& +\frac{1}{2}\left\{2 G\left(x_{1}+x_{4}\right)+I x_{5}+J x_{6}+(A+D) x_{7}+E x_{9}+F x_{10}\right\} z_{1} z_{4} \\
& +\frac{1}{2}\left\{2 H\left(x_{2}+x_{3}\right)+F x_{5}+E x_{6}+(B+C) x_{8}+J x_{9}+I x_{10}\right\} z_{2} z_{3} \\
& +\frac{1}{2}\left\{2 I\left(x_{2}+x_{4}\right)+G x_{5}+E x_{7}+J x_{8}+(B+D) x_{9}+H x_{10}\right\} z_{2} z_{4} \\
& +\frac{1}{2}\left\{2 J\left(x_{3}+x_{4}\right)+G x_{6}+F x_{7}+I x_{8}+H x_{9}+(C+D) x_{10}\right\} z_{3} z_{4} .
\end{aligned}
$$

We take a coordinate $(Y, V)$ in $\mathcal{P}_{4,1}$, that is,

$$
Y=\left(\begin{array}{cccc}
y_{1} & y_{5} & y_{6} & y_{7} \\
y_{5} & y_{2} & y_{8} & y_{9} \\
y_{6} & y_{8} & y_{3} & y_{10} \\
y_{7} & y_{9} & y_{10} & y_{4}
\end{array}\right) \quad \text { and } \quad V=\left(v_{1}, v_{2}, v_{3}, v_{4}\right)
$$

Put

$$
\frac{\partial}{\partial Y}=\left(\begin{array}{cccc}
\frac{\partial}{\partial y_{1}} & \frac{1}{2} \frac{\partial}{\partial y_{5}} & \frac{1}{2} \frac{\partial}{\partial y_{6}} & \frac{1}{2} \frac{\partial}{\partial y_{7}} \\
\frac{1}{2} \frac{\partial}{\partial y_{5}} & \frac{\partial}{\partial y_{2}} & \frac{1}{2} \frac{\partial}{\partial y_{8}} & \frac{1}{2} \frac{\partial}{\partial y_{9}} \\
\frac{1}{2} \frac{\partial}{\partial y_{6}} & \frac{1}{2} \frac{\partial}{\partial y_{8}} & \frac{\partial}{\partial y_{3}} & \frac{1}{2} \frac{\partial}{\partial y_{10}} \\
\frac{1}{2} \frac{\partial}{\partial y_{7}} & \frac{1}{2} \frac{\partial}{\partial y_{9}} & \frac{1}{2} \frac{\partial}{\partial y_{10}} & \frac{\partial}{\partial y_{4}}
\end{array}\right) \quad \text { and } \quad \frac{\partial}{\partial V}=\left(\frac{\partial}{\partial v_{1}}, \frac{\partial}{\partial v_{2}}, \frac{\partial}{\partial v_{3}}, \frac{\partial}{\partial v_{4}}\right) .
$$

Let

$$
D_{i}=\operatorname{tr}\left(\left(2 Y \frac{\partial}{\partial Y}\right)^{i}\right), \quad i=1,2,3,4
$$

and

$$
\Omega_{j}=\frac{\partial}{\partial V}\left(2 Y \frac{\partial}{\partial Y}\right)^{j} Y^{t}\left(\frac{\partial}{\partial V}\right), \quad j=0,1,2,3 .
$$

It is easily seen that

$$
D_{1}=\operatorname{tr}\left(2 Y \frac{\partial}{\partial Y}\right)=2 \sum_{i=1}^{10} y_{i} \frac{\partial}{\partial y_{i}}
$$

For brevity, we put

$$
\partial_{i}=\frac{\partial}{\partial v_{i}}, \quad i=1,2,3,4
$$

Then we get

$$
\begin{aligned}
\Omega_{0}= & y_{1} \partial_{1}^{2}+y_{2} \partial_{2}^{2}+y_{3} \partial_{3}^{2}+y_{4} \partial_{4}^{2}+2 y_{5} \partial_{1} \partial_{2} \\
& +2 y_{6} \partial_{1} \partial_{3}+2 y_{7} \partial_{1} \partial_{4}+2 y_{8} \partial_{2} \partial_{3}+2 y_{9} \partial_{2} \partial_{4}+2 y_{10} \partial_{3} \partial_{4} .
\end{aligned}
$$

We observe that $D_{1}, D_{2}, D_{3}, D_{4}, \Omega_{0}, \Omega_{1}, \Omega_{2}, \Omega_{3}$ are invariant differential operators in $\mathbb{D}\left(\mathcal{P}_{4,1}\right)$. Then we have the following relations

$$
\left[D_{i}, D_{j}\right]=0 \quad \text { for all } i, j=1,2,3,4
$$


and

$$
\left[D_{1}, \Omega_{0}\right]=2 \Omega_{0} .
$$

Therefore, $\mathbb{D}\left(\mathcal{P}_{4,1}\right)$ is not commutative.

\subsection{The case when $n=4$ and $m=2$}

In this case,

$G L_{4,2}=G L(4, \mathbb{R}) \ltimes \mathbb{R}^{(2,4)}, \quad K=O(4)$ and $\mathcal{P}_{4,2}=G L_{4,2} / K=\mathcal{P}_{4} \times \mathbb{R}^{(2,4)}$.

We see easily that

$$
\mathfrak{p}_{\star}=\left\{(X, Z) \mid X={ }^{t} X \in \mathbb{R}^{(4,4)}, \quad Z \in \mathbb{R}^{(2,4)}\right\} .
$$

Put

$$
\begin{aligned}
& E_{1}=\left(\begin{array}{llll}
1 & 0 & 0 & 0 \\
0 & 0 & 0 & 0 \\
0 & 0 & 0 & 0 \\
0 & 0 & 0 & 0
\end{array}\right), E_{2}=\left(\begin{array}{llll}
0 & 0 & 0 & 0 \\
0 & 1 & 0 & 0 \\
0 & 0 & 0 & 0 \\
0 & 0 & 0 & 0
\end{array}\right), E_{3}=\left(\begin{array}{llll}
0 & 0 & 0 & 0 \\
0 & 0 & 0 & 0 \\
0 & 0 & 1 & 0 \\
0 & 0 & 0 & 0
\end{array}\right), \\
& E_{4}=\left(\begin{array}{llll}
0 & 0 & 0 & 0 \\
0 & 0 & 0 & 0 \\
0 & 0 & 0 & 0 \\
0 & 0 & 0 & 1
\end{array}\right), E_{5}=\left(\begin{array}{llll}
0 & \frac{1}{2} & 0 & 0 \\
\frac{1}{2} & 0 & 0 & 0 \\
0 & 0 & 0 & 0 \\
0 & 0 & 0 & 0
\end{array}\right), E_{6}=\left(\begin{array}{llll}
0 & 0 & \frac{1}{2} & 0 \\
0 & 0 & 0 & 0 \\
\frac{1}{2} & 0 & 0 & 0 \\
0 & 0 & 0 & 0
\end{array}\right), \\
& E_{7}=\left(\begin{array}{llll}
0 & 0 & 0 & \frac{1}{2} \\
0 & 0 & 0 & 0 \\
0 & 0 & 0 & 0 \\
\frac{1}{2} & 0 & 0 & 0
\end{array}\right), E_{8}=\left(\begin{array}{llll}
0 & 0 & 0 & 0 \\
0 & 0 & \frac{1}{2} & 0 \\
0 & \frac{1}{2} & 0 & 0 \\
0 & 0 & 0 & 0
\end{array}\right), \\
& E_{9}=\left(\begin{array}{llll}
0 & 0 & 0 & 0 \\
0 & 0 & 0 & \frac{1}{2} \\
0 & 0 & 0 & 0 \\
0 & \frac{1}{2} & 0 & 0
\end{array}\right), E_{10}=\left(\begin{array}{llll}
0 & 0 & 0 & 0 \\
0 & 0 & 0 & 0 \\
0 & 0 & 0 & \frac{1}{2} \\
0 & 0 & \frac{1}{2} & 0
\end{array}\right) .
\end{aligned}
$$

Let $O_{4}$ be the $4 \times 4$ zero matrix and let

$$
O_{2,4}=\left(\begin{array}{cccc}
0 & 0 & 0 & 0 \\
0 & 0 & 0 & 0
\end{array}\right) \in \mathbb{R}^{(2,4)} .
$$

Put

$$
\begin{aligned}
e_{i} & =\left(E_{i}, O_{2,4}\right), \quad 1 \leq i \leq 10, \\
f_{1} & =\left(O_{4},\left(\begin{array}{llll}
1 & 0 & 0 & 0 \\
0 & 0 & 0 & 0
\end{array}\right)\right), f_{2}=\left(O_{4},\left(\begin{array}{llll}
0 & 1 & 0 & 0 \\
0 & 0 & 0 & 0
\end{array}\right)\right), \\
f_{3} & =\left(O_{4},\left(\begin{array}{llll}
0 & 0 & 1 & 0 \\
0 & 0 & 0 & 0
\end{array}\right)\right), f_{4}=\left(O_{4},\left(\begin{array}{llll}
0 & 0 & 0 & 1 \\
0 & 0 & 0 & 0
\end{array}\right)\right), \\
f_{5} & =\left(O_{4},\left(\begin{array}{llll}
0 & 0 & 0 & 0 \\
1 & 0 & 0 & 0
\end{array}\right)\right), f_{6}=\left(O_{4},\left(\begin{array}{llll}
0 & 0 & 0 & 0 \\
0 & 1 & 0 & 0
\end{array}\right)\right),
\end{aligned}
$$




$$
f_{7}=\left(O_{4},\left(\begin{array}{llll}
0 & 0 & 0 & 0 \\
0 & 0 & 1 & 0
\end{array}\right)\right), f_{8}=\left(O_{4},\left(\begin{array}{llll}
0 & 0 & 0 & 0 \\
0 & 0 & 0 & 1
\end{array}\right)\right) .
$$

Then $\left\{e_{i}, f_{j} \mid 1 \leq i \leq 10,1 \leq j \leq 8\right\}$ forms a basis for $\mathfrak{p}_{\star}$. Using this basis, we write for variables $(X, Z) \in \mathfrak{p}_{\star}$,

$$
X=\left(\begin{array}{cccc}
x_{1} & \frac{1}{2} x_{5} & \frac{1}{2} x_{6} & \frac{1}{2} x_{7} \\
\frac{1}{2} x_{5} & x_{2} & \frac{1}{2} x_{8} & \frac{1}{2} x_{9} \\
\frac{1}{2} x_{6} & \frac{1}{2} x_{8} & x_{3} & \frac{1}{2} x_{10} \\
\frac{1}{2} x_{7} & \frac{1}{2} x_{9} & \frac{1}{2} x_{10} & x_{4}
\end{array}\right) \quad \text { and } Z=\left(\begin{array}{cccc}
z_{11} & z_{12} & z_{13} & z_{14} \\
z_{21} & z_{22} & z_{23} & z_{24}
\end{array}\right)
$$

Set

$$
\begin{aligned}
& \square_{11}=\frac{1}{2}\left(2 A x_{1}+E x_{5}+F x_{6}+G x_{7}\right), \\
& \square_{22}=\frac{1}{2}\left(2 B x_{2}+E x_{5}+H x_{8}+I x_{9}\right), \\
& \square_{33}=\frac{1}{2}\left(2 C x_{3}+F x_{6}+H x_{8}+J x_{10}\right), \\
& \square_{44}=\frac{1}{2}\left(2 D x_{4}+G x_{7}+I x_{9}+J x_{10}\right), \\
& \square_{12}=\frac{1}{2}\left\{2 E\left(x_{1}+x_{2}\right)+(A+B) x_{5}+H x_{6}+I x_{7}+F x_{8}+G x_{9}\right\}, \\
& \square_{13}=\frac{1}{2}\left\{2 F\left(x_{1}+x_{3}\right)+H x_{5}+(A+C) x_{6}+J x_{7}+E x_{8}+G x_{10}\right\}, \\
& \square_{14}=\frac{1}{2}\left\{2 G\left(x_{1}+x_{4}\right)+I x_{5}+J x_{6}+(A+D) x_{7}+E x_{9}+F x_{10}\right\}, \\
& \square_{23}=\frac{1}{2}\left\{2 H\left(x_{2}+x_{3}\right)+F x_{5}+E x_{6}+(B+C) x_{8}+J x_{9}+I x_{10}\right\}, \\
& \square_{24}=\frac{1}{2}\left\{2 I\left(x_{2}+x_{4}\right)+G x_{5}+E x_{7}+J x_{8}+(B+D) x_{9}+H x_{10}\right\}, \\
& \square 34=\frac{1}{2}\left\{2 J\left(x_{3}+x_{4}\right)+G x_{6}+F x_{7}+I x_{8}+H x_{9}+(C+D) x_{10}\right\} .
\end{aligned}
$$

From Theorem 3.3, the algebra $\operatorname{Pol}\left(\mathfrak{p}_{\star}\right)^{K}$ is generated by the following 16 polynomials

$$
\begin{aligned}
\alpha_{1}(X, Z)= & x_{1}+x_{2}+x_{3}+x_{4}, \\
\alpha_{2}(X, Z)= & x_{1}^{2}+x_{2}^{2}+x_{3}^{2}+x_{4}^{2}+\frac{1}{2}\left(x_{5}^{2}+x_{6}^{2}+x_{7}^{2}+x_{8}^{2}+x_{9}^{2}+x_{10}^{2}\right), \\
\alpha_{3}(X, Z)= & x_{1}^{3}+x_{2}^{3}+x_{3}^{3}+x_{4}^{3} \\
& +\frac{3}{4} x_{1}\left(x_{5}^{2}+x_{6}^{2}+x_{7}^{2}\right)+\frac{3}{4} x_{2}\left(x_{5}^{2}+x_{8}^{2}+x_{9}^{2}\right) \\
& +\frac{3}{4} x_{3}\left(x_{6}^{2}+x_{8}^{2}+x_{10}^{2}\right)+\frac{3}{4} x_{4}\left(x_{7}^{2}+x_{9}^{2}+x_{10}^{2}\right) \\
& +\frac{3}{4}\left(x_{5} x_{6} x_{8}+x_{5} x_{7} x_{9}+x_{6} x_{7} x_{10}+x_{8} x_{9} x_{10}\right),
\end{aligned}
$$




$$
\begin{aligned}
& \alpha_{4}(X, Z)=A^{2}+B^{2}+C^{2}+D^{2}+2\left(E^{2}+F^{2}+G^{2}+H^{2}+I^{2}+J^{2}\right), \\
& \beta_{11}^{(0)}(X, Z)=z_{11}^{2}+z_{12}^{2}+z_{13}^{2}+z_{14}^{2} \text {, } \\
& \beta_{12}^{(0)}(X, Z)=z_{11} z_{21}+z_{12} z_{22}+z_{13} z_{23}+z_{14} z_{24}, \\
& \beta_{22}^{(0)}(X, Z)=z_{21}^{2}+z_{22}^{2}+z_{23}^{2}+z_{24}^{2} \text {, } \\
& \beta_{11}^{(1)}(X, Z)=x_{1} z_{11}^{2}+x_{2} z_{12}^{2}+x_{3} z_{13}^{2}+x_{4} z_{14}^{2}+x_{5} z_{11} z_{12} \\
& +x_{6} z_{11} z_{13}+x_{7} z_{11} z_{14}+x_{8} z_{12} z_{13}+x_{9} z_{12} z_{14}+x_{10} z_{13} z_{14}, \\
& \beta_{12}^{(1)}(X, Z)=x_{1} z_{11} z_{21}+x_{2} z_{12} z_{22}+x_{3} z_{13} z_{23}+x_{4} z_{14} z_{24} \\
& +\frac{1}{2} x_{5}\left(z_{11} z_{22}+z_{12} z_{21}\right)+\frac{1}{2} x_{6}\left(z_{11} z_{23}+z_{13} z_{21}\right) \\
& +\frac{1}{2} x_{7}\left(z_{11} z_{24}+z_{14} z_{21}\right)+\frac{1}{2} x_{8}\left(z_{12} z_{23}+z_{13} z_{22}\right) \\
& +\frac{1}{2} x_{9}\left(z_{12} z_{24}+z_{14} z_{22}\right)+\frac{1}{2} x_{10}\left(z_{13} z_{24}+z_{14} z_{23}\right), \\
& \beta_{22}^{(1)}(X, Z)=x_{1} z_{21}^{2}+x_{2} z_{22}^{2}+x_{3} z_{23}^{2}+x_{4} z_{24}^{2}+x_{5} z_{21} z_{22} \\
& +x_{6} z_{21} z_{23}+x_{7} z_{21} z_{23}++x_{8} z_{22} z_{23}+x_{9} z_{22} z_{24}+x_{10} z_{23} z_{24}, \\
& \beta_{11}^{(2)}(X, Z)=A z_{11}^{2}+B z_{12}^{2}+C z_{13}^{2}+D z_{14}^{2}+2 E z_{11} z_{12}+2 F z_{11} z_{13} \\
& +2 G z_{11} z_{14}+2 H z_{12} z_{13}+2 I z_{12} z_{14}+2 J z_{13} z_{14}, \\
& \beta_{12}^{(2)}(X, Z)=A z_{11} z_{21}+B z_{12} z_{22}+C z_{13} z_{23}+D z_{14} z_{24} \\
& +E\left(z_{11} z_{22}+z_{12} z_{21}\right)+F\left(z_{11} z_{23}+z_{13} z_{21}\right) \\
& +G\left(z_{11} z_{24}+z_{14} z_{21}\right)+H\left(z_{12} z_{23}+z_{13} z_{22}\right) \\
& +I\left(z_{12} z_{24}+z_{14} z_{22}\right)+J\left(z_{13} z_{24}+z_{14} z_{23}\right), \\
& \beta_{22}^{(2)}(X, Z)=A z_{21}^{2}+B z_{22}^{2}+C z_{23}^{2}+D z_{24}^{2}+2 E z_{21} z_{22}+2 F z_{21} z_{23} \\
& +2 G z_{21} z_{24}+2 H z_{22} z_{23}+2 I z_{22} z_{24}+2 J z_{23} z_{24} \text {, } \\
& \beta_{11}^{(3)}(X, Z)=\square_{11} z_{11}^{2}+\square_{22} z_{12}^{2}+\square_{33} z_{13}^{2}+\square_{44} z_{14}^{2}+\square_{12} z_{11} z_{12} \\
& +\square_{13} z_{11} z_{13}+\square_{14} z_{11} z_{14}+\square_{23} z_{12} z_{13} \\
& +\square_{24} z_{12} z_{14}+\square_{34} z_{13} z_{14} \text {, } \\
& \beta_{12}^{(3)}(X, Z)=\square_{11} z_{11} z_{21}+\square_{22} z_{12} z_{22}+\square_{33} z_{13} z_{23}+\square_{44} z_{14} z_{24} \\
& +\square_{12} z_{11} z_{22}+\square_{13} z_{11} z_{23}+\square_{14} z_{11} z_{24}+\square_{23} z_{12} z_{23} \\
& +\square_{24} z_{12} z_{24}+\square_{34} z_{13} z_{24} \text {, } \\
& \beta_{22}^{(3)}(X, Z)=\square_{11} z_{21}^{2}+\square_{22} z_{22}^{2}+\square_{33} z_{23}^{2}+\square_{44} z_{24}^{2}+\square_{12} z_{21} z_{22} \\
& +\square_{13} z_{21} z_{23}+\square_{14} z_{21} z_{24}+\square_{23} z_{22} z_{23} \\
& +\square_{24} z_{22} z_{24}+\square_{34} z_{23} z_{24} \text {. }
\end{aligned}
$$

Here, $A, B, C, \ldots, J$ are defined as in (7.1)-(7.10). 
Set

$$
\Delta_{a b}:=\operatorname{det}\left(\begin{array}{ll}
\beta_{11}^{(a)} & \beta_{12}^{(b)} \\
\beta_{12}^{(a)} & \beta_{22}^{(b)}
\end{array}\right) \quad \text { for } a, b=0,1,2,3 .
$$

By a tedious direct computation, we can show that

$$
\begin{aligned}
& \left(\alpha_{1}^{3}-3 \alpha_{1} \alpha_{2}+2 \alpha_{3}\right) \Delta_{00}-3\left(\alpha_{1}^{2}-\alpha_{2}\right)\left(\Delta_{01}+\Delta_{10}\right) \\
& +6 \alpha_{1}\left(\Delta_{02}+\Delta_{11}+\Delta_{20}\right)+6\left(\Delta_{03}+\Delta_{12}+\Delta_{21}+\Delta_{30}\right)=0 .
\end{aligned}
$$

Take a coordinate $(Y, V)$ in $\mathcal{P}_{4,2}$, that is,

$$
Y=\left(\begin{array}{lllc}
y_{1} & y_{5} & y_{6} & y_{7} \\
y_{5} & y_{2} & y_{8} & y_{9} \\
y_{6} & y_{8} & y_{3} & y_{10} \\
y_{7} & y_{9} & y_{10} & y_{4}
\end{array}\right) \quad \text { and } \quad V=\left(\begin{array}{cccc}
v_{11} & v_{12} & v_{13} & v_{14} \\
v_{21} & v_{22} & v_{23} & v_{24}
\end{array}\right)
$$

Put

$$
\frac{\partial}{\partial Y}=\left(\begin{array}{cccc}
\frac{\partial}{\partial y_{1}} & \frac{1}{2} \frac{\partial}{\partial y_{5}} & \frac{1}{2} \frac{\partial}{\partial y_{6}} & \frac{1}{2} \frac{\partial}{\partial y_{7}} \\
\frac{1}{2} \frac{\partial}{\partial y_{5}} & \frac{\partial}{\partial y_{2}} & \frac{1}{2} \frac{\partial}{\partial y_{8}} & \frac{1}{2} \frac{\partial}{\partial y_{9}} \\
\frac{1}{2} \frac{\partial}{\partial y_{6}} & \frac{1}{2} \frac{\partial}{\partial y_{8}} & \frac{\partial}{\partial y_{3}} & \frac{1}{2} \frac{\partial}{\partial y_{10}} \\
\frac{1}{2} \frac{\partial}{\partial y_{7}} & \frac{1}{2} \frac{\partial}{\partial y_{9}} & \frac{1}{2} \frac{\partial}{\partial y_{10}} & \frac{\partial}{\partial y_{4}}
\end{array}\right) \text { and } \frac{\partial}{\partial V}=\left(\begin{array}{llll}
\frac{\partial}{\partial v_{11}} & \frac{\partial}{\partial v_{12}} & \frac{\partial}{\partial v_{13}} & \frac{\partial}{\partial v_{14}} \\
\frac{\partial}{\partial v_{21}} & \frac{\partial}{\partial v_{22}} & \frac{\partial}{\partial v_{23}} & \frac{\partial}{\partial v_{24}}
\end{array}\right) .
$$

Let

and

$$
D_{i}=\operatorname{tr}\left(\left(2 Y \frac{\partial}{\partial Y}\right)^{i}\right), \quad i=1,2,3,4
$$

$$
\Omega_{p q}^{(k)}=\left\{\frac{\partial}{\partial V}\left(2 Y \frac{\partial}{\partial Y}\right)^{k} Y^{t}\left(\frac{\partial}{\partial V}\right)\right\}_{p q}, \quad k=0,1,2,3,1 \leq p \leq q \leq 2 .
$$

Note that $D_{1}, D_{2}, D_{3}, D_{4}, \Omega_{11}^{(0)}, \ldots, \Omega_{22}^{(3)}$ are $G L_{4,2}$-invariant. It is easily seen that

$$
D_{1}=\operatorname{tr}\left(2 Y \frac{\partial}{\partial Y}\right)=2 \sum_{i=1}^{10} y_{i} \frac{\partial}{\partial y_{i}}
$$

For brevity, we put

$$
\partial_{i j}=\frac{\partial}{\partial v_{i j}}, \quad i=1,2,1 \leq j \leq 4
$$

Then we get

$$
\begin{aligned}
\Omega_{11}^{(0)}= & y_{1} \partial_{11}^{2}+y_{2} \partial_{12}^{2}+y_{3} \partial_{13}^{2}+y_{4} \partial_{14}^{2}+2 y_{5} \partial_{11} \partial_{12}+2 y_{6} \partial_{11} \partial_{13} \\
& +2 y_{7} \partial_{11} \partial_{14}+2 y_{8} \partial_{12} \partial_{13}+2 y_{9} \partial_{12} \partial_{14}+2 y_{10} \partial_{13} \partial_{14}, \\
\Omega_{12}^{(0)}= & y_{1} \partial_{11} \partial_{21}+y_{2} \partial_{12} \partial_{22}+y_{3} \partial_{13} \partial_{23}+y_{4} \partial_{14} \partial_{24} \\
& +y_{5}\left(\partial_{11} \partial_{22}+\partial_{12} \partial_{21}\right)+y_{6}\left(\partial_{11} \partial_{23}+\partial_{13} \partial_{21}\right) \\
& +y_{7}\left(\partial_{11} \partial_{24}+\partial_{14} \partial_{21}\right)+y_{8}\left(\partial_{12} \partial_{23}+\partial_{13} \partial_{22}\right)
\end{aligned}
$$




$$
\begin{gathered}
+y_{9}\left(\partial_{12} \partial_{24}+\partial_{14} \partial_{22}\right)+y_{10}\left(\partial_{13} \partial_{24}+\partial_{14} \partial_{23}\right) \\
\Omega_{22}^{(0)}= \\
y_{1} \partial_{21}^{2}+y_{2} \partial_{22}^{2}+y_{3} \partial_{23}^{2}+y_{4} \partial_{24}^{2}+2 y_{5} \partial_{21} \partial_{22}+2 y_{6} \partial_{21} \partial_{23} \\
+2 y_{7} \partial_{21} \partial_{24}+2 y_{8} \partial_{22} \partial_{23}+2 y_{9} \partial_{22} \partial_{24}+2 y_{10} \partial_{23} \partial_{24} .
\end{gathered}
$$

Then we have the following relations

$$
\left[D_{i}, D_{j}\right]=0 \quad \text { for all } i, j=1,2,3,4 \text {, }
$$$$
\left[\Omega_{k l}^{(0)}, \Omega_{p q}^{(0)}\right]=0, \quad 1 \leq k \leq l \leq 2,1 \leq p \leq q \leq 2,
$$

and

$$
\left[D_{1}, \Omega_{11}^{(0)}\right]=2 \Omega_{11}^{(0)}, \quad\left[D_{1}, \Omega_{12}^{(0)}\right]=2 \Omega_{12}^{(0)}, \quad\left[D_{1}, \Omega_{22}^{(0)}\right]=2 \Omega_{22}^{(0)} .
$$

Therefore, $\mathbb{D}\left(\mathcal{P}_{4,2}\right)$ is not commutative.

\section{Final remarks}

In this section, we present some open problems and discuss a notion of automorphic forms on $\mathcal{P}_{n, m}$ using $G L_{n, m}$-invariant differential operators on the Minkowski-Euclid space $\mathcal{P}_{n, m}$.

Recall the invariant polynomials $\alpha_{j}(1 \leq j \leq n)$ from (3.11) and $\beta_{p q}^{(k)}(0 \leq$ $k \leq n-1,1 \leq p \leq q \leq m)$ from (3.12). Also recall the invariant differential operators $D_{j}(1 \leq j \leq n)$ from (3.19) and $\Omega_{p q}^{(k)}(0 \leq k \leq n-1,1 \leq p \leq q \leq m)$ from (3.20).

Theorem 8.1. The following relations hold:

$$
\left[\Omega_{k l}^{(0)}, \Omega_{p q}^{(0)}\right]=0, \quad 1 \leq k \leq l \leq m, 1 \leq p \leq q \leq m,
$$

$$
\left[D_{1}, \Omega_{p q}^{(0)}\right]=2 \Omega_{p q}^{(0)} \quad \text { for all } 1 \leq p \leq q \leq m .
$$

Proof. The relation (8.1) follows from the work of Atle Selberg (cf. [8, 10, 11]). Take a coordinate $(Y, V)$ in $\mathcal{P}_{n, m}$ with $Y=\left(y_{i j}\right)$ and $V=\left(v_{k l}\right)$. Put

$$
\frac{\partial}{\partial Y}=\left(\frac{1+\delta_{i j}}{2} \frac{\partial}{\partial y_{i j}}\right) \text { and } \frac{\partial}{\partial V}=\left(\frac{\partial}{\partial v_{k l}}\right),
$$

where $1 \leq i, j, l \leq n$ and $1 \leq k \leq m$. Then we get

$$
\begin{aligned}
D_{1} & =2 \sum_{1 \leq i \leq j \leq n} y_{i j} \frac{\partial}{\partial y_{i j}}, \\
\Omega_{p q}^{(0)} & =\sum_{a=1}^{n} y_{a a} \frac{\partial^{2}}{\partial v_{p a} \partial v_{q a}}+\sum_{1 \leq a<b \leq n} y_{a b}\left(\frac{\partial^{2}}{\partial v_{p a} \partial v_{q b}}+\frac{\partial^{2}}{\partial v_{p b} \partial v_{q a}}\right) .
\end{aligned}
$$

By a direct calculation, we obtain the desired relations (8.2) and (8.3). 


\section{Conjecture 2.}

$$
\begin{aligned}
\text { (8.4) } \quad \Theta_{n, m}\left(\alpha_{j}\right) & =D_{j} \quad \text { for all } 1 \leq j \leq n, \\
\text { (8.5) } \quad \Theta_{n, m}\left(\beta_{p q}^{(k)}\right) & =\Omega_{p q}^{(k)} \quad \text { for all } 0 \leq k \leq n-1,1 \leq p \leq q \leq m .
\end{aligned}
$$

We refer to Conjecture 1 in Section 2.

Conjecture 3. The invariants $D_{j}(1 \leq j \leq n)$ and $\Omega_{p q}^{(k)}(0 \leq k \leq n-1,1 \leq$ $p \leq q \leq m)$ generate the noncommutative algebra $\mathbb{D}\left(\mathcal{P}_{n, m}\right)$.

Conjecture 4. The above relations (8.1), (8.2) and (8.3) generate all relations among the set

$$
\left\{D_{j}, \Omega_{p q}^{(k)} \mid 1 \leq j \leq n, 0 \leq k \leq n-1,1 \leq p \leq q \leq m\right\} .
$$

Problem 8. Find a natural way to construct generators of $\mathbb{D}\left(\mathcal{P}_{n, m}\right)$.

Using $G L_{n, m}$-invariant differential operators on the Minkowski-Euclid space $\mathcal{P}_{n, m}$, we introduce a notion of automorphic forms on $\mathcal{P}_{n, m}$ (cf. [11]).

Let

$$
\Gamma_{n, m}:=G L(n, \mathbb{Z}) \ltimes \mathbb{Z}^{(m, n)}
$$

be the arithmetic subgroup of $G L_{n, m}$. Let $\mathcal{Z}_{n, m}$ be the center of $\mathbb{D}\left(\mathcal{P}_{n, m}\right)$.

Definition 8.1. A smooth function $f: \mathcal{P}_{n, m} \longrightarrow \mathbb{C}$ is said to be an automorphic form for $\Gamma_{n, m}$ if it satisfies the following conditions:

(A1) $f$ is $\Gamma_{n, m}$-invariant.

(A2) $f$ is an eigenfunction of any differential operator in the center $\mathcal{Z}_{n, m}$ of $\mathbb{D}\left(\mathcal{P}_{n, m}\right)$.

(A3) $f$ has a growth condition.

We define another notion of automorphic forms as follows.

Definition 8.2. Let $\mathbb{D}_{\text {a }}$ be a commutative subalgebra of $\mathbb{D}\left(\mathcal{P}_{n, m}\right)$ containing the Laplacian $\Delta_{n, m ; A, B}$. A smooth function $f: \mathcal{P}_{n, m} \longrightarrow \mathbb{C}$ is said to be an automorphic form for $\Gamma_{n, m}$ with respect to $\mathbb{D}_{\bullet}$ if it satisfies the following conditions:

(A1) $f$ is $\Gamma_{n, m}$-invariant.

(A2) $f$ is an eigenfunction of any differential operator in $\mathbb{D}$

(A3) $f$ has a growth condition.

\section{References}

[1] Harish-Chandra, Representations of semisimple Lie groups. I, Trans. Amer. Math. Soc. 75 (1953), 185-243.

[2] — The characters of semisimple Lie groups, Trans. Amer. Math. Soc. 83 (1956), 98-163.

[3] S. Helgason, Differential operators on homogeneous spaces, Acta Math. 102 (1959), 239-299.

[4] , Groups and Geometric Analysis, Academic Press, New York, 1984. 
[5] R. Howe, Perspectives on invariant theory: Schur duality, multiplicity-free actions and beyond, The Schur lectures (1992) (Tel Aviv), 1-82, Israel Math. Conf. Proc., 8, Bar-Ilan Univ., Ramat Gan, 1995.

[6] M. Itoh, On the Yang Problem (SFT), Max-Planck Institut für Mathematik, Bonn, 2011.

[7] H. Maass, Die Bestimmung der Dirichletreihnen mit Grössencharakteren zu den Modulformen n-ten Grades, J. Indian Math. Soc. 9 (1955), 1-23.

[8] _ Siegel modular forms and Dirichlet series, Lecture Notes in Math., vol. 216, Springer-Verlag, Berlin-Heidelberg-New York, 1971.

[9] H. Minkowski, Gesammelte Abhandlungen, Chelsea, New York, 1967.

[10] A. Selberg, Harmonic analysis and discontinuous groups in weakly symmetric Riemannian spaces with applications to Dirichlet series, J. Indian Math. Soc. B. 20 (1956), 47-87.

[11] A. Terras, Harmonic Analysis on Symmetric Spaces and Applications II, SpringerVerlag, 1988.

[12] H. Weyl, The classical groups: Their invariants and representations, Princeton Univ. Press, Princeton, New Jersey, second edition, 1946.

[13] J.-H. Yang, Singular Jacobi forms, Trans. Amer. Math. Soc. 347 (1995), no. 6, 20412049.

[14] , Polarized Real Tori, arXiv:0912.5084v1 [math.AG] (2009) or a revised version (2012).

Department of Mathematics

INHA UNIVERSITY

INCHEON 402-751, KOREA

E-mail address: jhyang@inha.ac.kr 